MADPH-00-1195

OKHEP-00-08

hep-ph/0105182

May 2001

\title{
Indirect Search for Neutralino Dark Matter with High Energy Neutrinos
}

\author{
V. Barger ${ }^{a}$, Francis Halzen ${ }^{a}$, Dan Hooper ${ }^{a}$ and Chung $\mathrm{Kao}^{b}$ \\ ${ }^{a}$ Department of Physics, University of Wisconsin, Madison, WI 53706 \\ ${ }^{b}$ Department of Physics and Astronomy, University of Oklahoma, Norman, OK 73019
}

\begin{abstract}
We investigate the prospects of indirect searches for supersymmetric neutralino dark matter. Relic neutralinos gravitationally accumulate in the Sun and their annihilations produce high energy neutrinos. Muon neutrinos of this origin can be seen in large detectors like AMANDA, IceCube and ANTARES. We evaluate the relic density and the detection rate in several models - the minimal supersymmetric model, minimal supergravity, and supergravity with non-universal Higgs boson masses at the grand unification scale. We make realistic estimates for the indirect detection rates including effects of the muon detection threshold, quark hadronization, and solar absorption. We find good prospects for detection of neutralinos with mass above $200 \mathrm{GeV}$.
\end{abstract}




\section{INTRODUCTION}

Average matter and energy densities in the Universe $\left(\rho_{i}\right)$ are commonly described in terms of density parameters $\left(\Omega_{i}=\rho_{i} / \rho_{c}\right)$, where

$$
\rho_{c}=3 H_{0}^{2} /\left(8 \pi G_{N}\right) \simeq 1.88 \times 10^{-29} h^{2} \mathrm{~g} / \mathrm{cm}^{3},
$$

is the critical density to close the Universe, $h=H_{0} /\left(100 \mathrm{~km} \mathrm{sec}^{-1} \mathrm{Mpc}^{-1}\right), H_{0}$ is the Hubble constant, and $G_{N}$ is Newton's gravitational constant. Recent measurements of the Hubble constant are converging to $h \simeq 0.6-0.7$ [迆].

Studies of clusters of galaxies and large scale structure indicate that the matter density should be at least $\Omega_{M} \gtrsim 0.2$ [4]. The matter density inferred from observations of cluster Xray [5], supernovae [6], and the cosmic microwave background radiation (CMB) anisotropy data [7] is $\Omega_{M} \simeq 0.3-0.4$. The baryon density inferred from Big-Bang Nucleosynthesis is $\Omega_{b} h^{2} \simeq 0.020 \pm 0.002$ [10] which is in good agreement with the value obtained from recent analyses of the CMB power spectrum [7,9.9. The luminous matter in the Universe has a very small relative density $\Omega_{\mathrm{L}} \lesssim 0.01$ [11]. Therefore, the evidence for substantial dark matter is compelling. Moreover, the dominant component must be cold dark matter to be consistent with large scale structure [12]. The most attractive cold dark matter candidates are stable weakly interacting mass particles (WIMPs) produced in the early Universe. In our analysis, we take $\Omega_{\chi}=\Omega_{M}-\Omega_{b}$ to be the WIMP density and conservatively consider

$$
0.05 \lesssim \Omega_{\chi} h^{2} \lesssim 0.3
$$

as the cosmologically interesting region for the cold dark matter density .

In the early Universe, the temperature $(T)$ was much higher than the WIMP mass $\left(m_{\chi}\right)$ and WIMPs existed abundantly in thermal equilibrium with WIMP annihilation into lighter particles balanced by pair production. When the Universe cooled and the temperature fell below $m_{\chi}$, the WIMP density became suppressed by the Boltzmann factor $\exp \left(-m_{\chi} / T\right)$. As a result of the expansion of the Universe, WIMPs dropped out of equilibrium with other particles at some point, leaving a relic abundance.

From simple dimensional analysis, the WIMP annihilation cross section is about

$$
\sigma \sim \alpha^{2} / m_{\chi}^{2}
$$

and their relative density is

$$
\Omega_{\chi} h^{2}=m_{\chi} n_{\chi} / \rho_{c} \simeq\left(3 \times 10^{-27} \mathrm{~cm}^{3} / \mathrm{s}\right) /<\sigma \mathrm{v}>\sim\left(3 \times 10^{-27} \mathrm{~cm}^{3} / \mathrm{s}\right)\left(\mathrm{m}_{\chi}^{2} / \alpha^{2}\right),
$$

where $\alpha$ is the fine-structure constant. For a WIMP mass of the order of the weak boson mass, $m_{\chi} \sim m_{W}$, the WIMP density is $\Omega_{\chi} \sim 1$. Thus, a cosmologically interesting $\Omega_{\chi}$ is natural in theories with a weak scale WIMP mass.

In supersymmetric (SUSY) theories with a conserved $R$-parity円, the lightest SUSY particle (LSP) is stable and is thus an attractive WIMP candidate [14] 16. Most commonly, the

\footnotetext{
${ }^{1} R=+1$ for particles in the Standard Model as well as Higgs bosons and $R=-1$ for their superpartners.
} 
LSP is the lightest neutralino which is a superposition of the supersymmetric partners of the photon, the $Z$ boson and the neutral Higgs bosons. The gaugino and higgsino composition of the neutralino is an important factor in the neutralino annihilation cross section. If the neutralinos are more gaugino-like, they annihilate mostly into $b \bar{b}$ and $\tau \bar{\tau}$; if the neutralinos are higgsino-like, most of them annihilate into gauge bosons and $t \bar{t}$.

WIMPs in the halo of our galaxy lose energy when they pass through the Sun and scatter from nuclei. When their velocities fall below the escape velocity from the Sun, they become gravitationally trapped and accumulate. Annihilations of these WIMPs are a potential source of high energy neutrinos that can be detected via Cerenkov light by large under-ice or under-water photomultiplier arrays in neutrino telescopes, such as AMANDA [17], IceCube [18] and ANTARES [19].

In this paper we investigate the prospects of observing muon neutrinos produced by WIMP annihilation in the Sun. Due to cosmic ray backgrounds, only the signals from the muon neutrinos at or below the horizontal detection can be detected. In Section II, we estimate the general expectations for neutrino signals from WIMP annihilation, the effect of the muon detection energy threshold, and the backgrounds to the signal. In Sections III, IV and V, we evaluate the indirect detection rate within the framework of the minimal supersymmetric model (MSSM), the minimal supergravity model (mSUGRA), and supergravity unified models with non-universal Higgs boson masses at the grand unified scale, respectively. In Section VI and VII, we summarize the prospects of indirect search and our conclusions about indirect detection of neutralino dark matter in large ice or water experiments.

\section{INDIRECT DETECTION OF WIMPS}

When our galaxy formed, the cold dark matter WIMPs were clustered with the luminous matter and formed a significant fraction of the galactic matter density [20,21]

$$
\rho_{\chi} \simeq 0.3-0.5 \mathrm{GeV} / \mathrm{cm}^{3}
$$

as deduced from rotation curves. The distribution of WIMPs in the galactic halo is usually approximated as an isothermal sphere with an average velocity [22 24]

$$
v_{\chi} \simeq 220-300 \mathrm{~km} / \mathrm{sec} .
$$

The density of WIMPs that are gravitationally trapped in the Sun builds up until equilibrium is reached when their annihilation rate is half of the capture rate (the factor of 2 is due to the Majorana nature of WIMPs). Since the WIMP mass is naturally in the $100 \mathrm{GeV}-1000 \mathrm{GeV}$ mass range, they annihilate mostly into weak bosons and heavy quarks, which subsequently decay into final states with high-energy neutrinos [16,25]. The high-energy muon neutrinos can be detected in large ice or water detectors by the Cherenkov light from the muons. The muons in these events are aligned along a direction pointing back to the Sun. The angular

spread of the muons from this direction is $\theta_{\mu} \sim 1.2^{\circ} / \sqrt{E_{\mu}(\mathrm{TeV})}$. The atmospheric neutrino events in a pixel containing this signal are the backgrounds. The measurement of muon energy might be used to (i) focus the search window around the direction from the Sun in order to reduce the background and (ii) estimate the neutrino energy from the angular spread. 


\section{A. Indirect Detection Rate}

In this subsection, we make a rough estimate of the indirect detection rate for a generic WIMP dark matter under the following assumptions] [25]:

(i) the measured galactic halo density is mostly associated with WIMPs and their flux is

$$
\begin{aligned}
\phi_{\chi} & =n_{\chi} v_{\chi} \simeq \frac{0.4 \mathrm{~cm}^{-3}}{m_{\chi}(\mathrm{GeV})} \times\left(3 \times 10^{7} \mathrm{~cm} / \mathrm{s}\right) \\
& \simeq \frac{1.2 \times 10^{7}}{m_{\chi}(\mathrm{GeV})} \mathrm{cm}^{-2} \mathrm{~s}^{-1}
\end{aligned}
$$

where $m_{\chi}$ is the WIMP mass;

(ii) the WIMP-nucleon cross section is approximately given by the dimensional analysis result

$$
\sigma(\chi N) \equiv \sigma_{\mathrm{DA}} \simeq\left(G_{F} m_{N}^{2}\right)^{2} \frac{1}{m_{W}^{2}}=6 \times 10^{-42} \mathrm{~cm}^{2}
$$

(iii) the WIMPs annihilate about $10 \%$ of the time into neutrinos via the channels $\chi \bar{\chi} \rightarrow$ $W^{+} W^{-}$or $Q \bar{Q}$ where $Q$ is a heavy quark (bottom or top).

The further physical quantities needed to calculate the indirect detection rate for neutrinos from WIMP annihilation in the Sun are as follows:

(i) the capture cross section in the Sun $\left(\sigma_{\odot}\right)$, given by the product of the number of target nucleons in the Sun and the elastic $\chi N$ cross section $\left(\sigma_{E l}\right)$ and a focusing factor $(f \simeq 10)$ that is given by the ratio of kinetic and potential energy of the WIMP near the Sun [26].

$$
\sigma_{\odot}=f\left[1.2 \times 10^{57}\right] \sigma_{\mathrm{El}}
$$

(ii) the neutrino flux at the Earth from WIMP annihilation in the Sun

$$
\phi_{\nu}=B_{\chi} \phi_{\chi} \sigma_{\odot} / 4 \pi d^{2}
$$

where $d=1$ A.U. $=1.5 \times 10^{13} \mathrm{~cm}$ and $B_{\chi}$ is the branching fraction to neutrinos $(\sim 10 \%)$. Thus we find

$$
\phi_{\nu}=\frac{3 \times 10^{-5}}{m_{\chi}(\mathrm{GeV})} \mathrm{cm}^{-2} \mathrm{~s}^{-1}
$$

\footnotetext{
${ }^{2}$ In this section we take $\rho_{\chi}=0.4 \mathrm{GeV} / \mathrm{cm}^{3}$ and $v_{\chi}=300 \mathrm{~km} / \mathrm{s}$.
} 
(iii) the probability of neutrino detection [26], given by

$$
P=N_{A} \sigma_{\nu} R_{\mu} \simeq 2 \times 10^{-13}\left[m_{\chi}(\mathrm{GeV})\right]^{2},
$$

where $N_{A} \simeq 6 \times 10^{23}$ is the Avogadro number, $\sigma_{\nu}=0.5 \times 10^{-38} E_{\nu}(\mathrm{GeV}) \mathrm{cm}^{2}$ is the average of the $\nu N$ and $\bar{\nu} N$ cross sections, and $R_{\mu} \simeq 500 \mathrm{~cm} \times \mathrm{E}_{\mu}(\mathrm{GeV})$ is the muon range. For the kinematics of the annihilation and decay chain

$$
\begin{aligned}
\chi \bar{\chi} & \rightarrow W^{+} W^{-} \\
\stackrel{\longrightarrow}{\rightarrow} \mu \nu_{\mu} &
\end{aligned}
$$

the average neutrino energy is about $<E_{\nu}>\simeq \frac{1}{2} m_{\chi}$. For the decay chain into heavy quarks

$$
\chi \bar{\chi} \rightarrow Q \bar{Q} \underset{\hookrightarrow q \mu \nu_{\mu}}{ }
$$

the average neutrino energy is about $<E_{\nu}>\simeq \frac{1}{3} m_{\chi}$.

(iv) the number of neutrino events per year per unit area $\left(\mathrm{m}^{2}\right)$ detectable in a neutrino telescope from $\chi \bar{\chi}$ annihilation in the Sun

$$
d N_{\mathrm{ID}} / d A=\phi_{\nu} P \simeq 2 \times 10^{-6} m_{\chi}(\mathrm{GeV})(\text { year })^{-1}\left(\mathrm{~m}^{2}\right)^{-1} .
$$

For an effective area of $10^{4} \mathrm{~m}^{2}$, the indirect detection rate is approximately

$$
d N_{\mathrm{ID}} / d A \simeq 2 \times 10^{-2} m_{\chi}(\mathrm{GeV})\left(10^{4} \mathrm{~m}^{2}\right)^{-1}(\text { year })^{-1} .
$$

\section{B. Neutrino-generated Muons}

Neutrino telescopes detect muons from the charged-current reaction

$$
\nu_{\mu}+N \rightarrow \mu+X,
$$

where $N$ is an average nucleon in the Earth below the detector. The effective detection volume is the product of the detector area and the muon range in rock $\left(R_{\mu}\right)$ which increases with energy. By observing muons produced by charged current of $\nu_{\mu}\left(\bar{\nu}_{\mu}\right)$ interactions in the rock below the detector, the effective volume is enhanced. The characteristic range of $\mathrm{TeV}$ muons is about one kilometer in rock, so large effective volumes are realized at $\mathrm{TeV}$ energies. This technique applies only for upward going muons entering the detector from below the horizontal, because of the large backgrounds from atmospheric muons that dominate neutrino signals from above the horizontal.

The average muon energy loss rate is

$$
\left\langle\frac{\mathrm{d} E}{\mathrm{~d} X}\right\rangle=-\alpha(E)-\beta(E) \times E,
$$


where $X$ is the thickness of material in $\mathrm{g} / \mathrm{cm}^{2}$. The first term represents ionization losses, which are approximately continuous, with $\alpha \sim 2 \mathrm{MeV} \mathrm{g} \mathrm{g}^{-1} \mathrm{~cm}^{2}$. The second term includes the catastrophic processes of bremsstrahlung, pair production and nuclear interactions, in which fluctuations play an essential role. Here $\beta \sim 4 \times 10^{-6} \mathrm{~g}^{-1} \mathrm{~cm}^{2}$. The critical energy above which the radiative processes dominate is

$$
E_{\text {cr }}=\alpha / \beta \approx 500 \mathrm{GeV} \text {. }
$$

To treat muon propagation properly when $E_{\mu}>E_{\text {cr }}$ requires a Monte Carlo calculation of the probability $P_{\text {surv }}$ that a muon of energy $E_{\mu}$ survives with energy $>E_{\mu}^{\text {min }}$ after propagating a distance $X$ [27]. The probability that a neutrino of energy $E_{\nu}$ on a trajectory through a detector produces a muon above the threshold energy at the detector is [27 29]

$$
P_{\nu}\left(E_{\nu}, E_{\mu}^{\min }\right)=N_{A} \int_{0}^{E_{\nu}} \mathrm{d} E_{\mu} \frac{\mathrm{d} \sigma_{\nu}}{\mathrm{d} E_{\mu}}\left(E_{\mu}, E_{\nu}\right) R_{\mathrm{eff}}\left(E_{\mu}, E_{\mu}^{\min }\right)
$$

where

$$
R_{\text {eff }}=\int_{0}^{\infty} \mathrm{d} X P_{\text {surv }}\left(E_{\mu}, E_{\mu}^{\min }, X\right) .
$$

The flux of $\nu_{\mu}$-generated muons at the detector is given by a convolution of the neutrino spectrum $\phi_{\nu}$ with the muon production probability (18) as

$$
\phi_{\mu}\left(E_{\mu}^{\min }, \theta\right)=\int_{E_{\mu}^{\min }} \mathrm{d} E_{\nu} P_{\nu}\left(E_{\nu}, E_{\mu}^{\min }\right) \exp \left[-\sigma_{\text {tot }}\left(E_{\nu}\right) N_{A} X(\theta)\right] \phi_{\nu}\left(E_{\nu}, \theta\right) .
$$

The exponential factor in the integrand accounts for absorption of neutrinos along the chord through the Earth, $X(\theta)$. Absorption becomes important for $\sigma\left(E_{\nu}\right) \gtrsim 10^{-33} \mathrm{~cm}^{2}$ or $E_{\nu} \gtrsim$ $10^{7} \mathrm{GeV}$. The event rate is calculated by multiplying Eq. (20) with the effective area of the detector.

The probability $P_{\nu}\left(E_{\nu}, 0\right)$ can be approximated with a power law

$$
P_{\nu \rightarrow \mu} \simeq 1.3 \times 10^{-6}\left(\frac{E}{\mathrm{TeV}}\right)^{2.2}
$$

for $E_{\nu}$ between $1 \mathrm{GeV}$ and $1 \mathrm{TeV}$. This energy dependence reflects the neutrino cross section $\left(\sigma_{\nu} \propto E\right)$ and the effective muon range $\left(R_{\mu} \propto E\right)$ in Eq. (18). Above $1 \mathrm{TeV}$, the effect of the $W$ propagator slows the growth of $\sigma_{\nu}$ and the muon range also makes a transition from linearity to a more gradual energy dependence.

\section{Atmospheric Neutrino Backgrounds}

The background to indirect detection (ID) is determined by the flux of atmospheric neutrinos in a pixel region of the detector that subtends the Sun. The number of upward atmospheric background events per year in a $10^{4} \mathrm{~m}^{2}$ detector is $\sim 10^{2} / E_{\mu}(\mathrm{TeV})$. The size of the pixel region is determined by the angle between muon and neutrino $\left(\sim 1.2^{\circ} / \sqrt{E_{\mu}(\mathrm{TeV})}\right)$. Using the kinematics $E_{\mu} \simeq m_{\chi} / 4$ for the WIMP signal from annihilation to $W$-pairs, we obtain 


$$
B_{\mathrm{ID}}=\frac{10^{2} / E_{\mu}(\mathrm{TeV})}{2 \pi /\left[\frac{1.2^{\circ} \frac{\pi}{180^{\circ}}}{\sqrt{E_{\mu}(\mathrm{TeV})}}\right]^{2}}=\frac{1.1 \times 10^{5}}{m_{\chi}^{2}(\mathrm{GeV})^{2}} 10^{4} \mathrm{~m}^{2-1} \text { year }^{-1}
$$

This expression is only valid for for $E_{\mu} \approx m_{\chi} / 4>100 \mathrm{GeV}$. More generally, the backgrounds are given in Table I.

TABLE I. Number of background events due to atmospheric neutrinos.

\begin{tabular}{|c|c|c|c|}
\hline$\underline{E_{\mu}(\mathrm{GeV})}$ & $\begin{array}{l}\mathrm{N}(\text { Background }) \\
\text { in } 10^{4} \mathrm{~m}^{2}\end{array}$ & $\begin{array}{l}\text { Number of pixels } \\
\text { of solar size } \\
\text { in } 2 \pi \\
\end{array}$ & $\begin{array}{c}\mathrm{N}(\text { Background }) \\
\text { per } 10^{4} \mathrm{~m}^{2} \\
\text { per pixel, per year }\end{array}$ \\
\hline 10 & 3200 & 140 & 23 \\
\hline 100 & 1060 & $1.4 \times 10^{3}$ & 0.8 \\
\hline 1000 & 110 & $1.4 \times 10^{4}$ & $8 \times 10^{-3}$ \\
\hline
\end{tabular}

For large $m_{\chi}$ the signal to background ratio is

$$
\left(\frac{N}{B}\right)_{\mathrm{ID}} \equiv \frac{d N_{\mathrm{ID}} / d A}{d B_{\mathrm{ID}} / d A} \simeq 7.2 \times 10^{-6} m_{\chi}^{3}(\mathrm{GeV})^{3}
$$

Table II shows the expected signal and signal to background estimates for several values of $m_{\chi}$. Thus high-energy muons pointing at the Sun are a viable signal for sufficiently high $m_{\chi}$.

TABLE II. Event rates and signal to background $\left(N_{S} / N_{B}\right)$ ratio for an effective detector area of $10^{4} \mathrm{~m}^{2}$ in a year.

\begin{tabular}{c|cc}
\hline \hline$m_{\chi}(\mathrm{GeV})$ & Indirect detection rate $\left(\right.$ events $/ 10^{4} \mathrm{~m}^{2} /$ year $)$ & $N_{S} / N_{B}$ \\
\hline 50 & $2.3 \times 10^{1}$ & $\simeq 1$ \\
500 & $2 \times 10^{2}$ & $\simeq 10^{2}$ \\
2000 & $1.7 \times 10^{2}$ & $\simeq 10^{4}$ \\
\hline \hline
\end{tabular}

In the next three sections we make quantitative calculations of the WIMP annihilation rates expected in supersymmetry and supergravity models to replace the simple estimate from Eq.(8).

\section{THE MINIMAL SUPERSYMMETRIC MODEL}

A supersymmetry (SUSY) between fermions and bosons is a compelling extension of the Standard Model (SM) for several reasons.

(i) It provides an elegant solution to the fine tuning problem;

(ii) With the particle mass spectra of the minimal supersymmetric standard model (MSSM) the evolution of renormalization group equations is consistent with a grand unified scale $M_{\mathrm{GUT}} \sim 2 \times 10^{16} \mathrm{GeV}$ and a SUSY mass scale in the range $M_{Z} \lesssim M_{\mathrm{SUSY}} \lesssim 1 \mathrm{TeV}$;

(iii) With a large top quark Yukawa coupling at a grand unified scale $\left(M_{\mathrm{GUT}}\right)$, radiative corrections can drive a Higgs boson mass squared parameter negative, spontaneously breaking the electroweak symmetry, and naturally explain the origin of the electroweak scale; 
(iv) If $R$-parity $f^{5}$ is conserved, the lightest neutralino in supersymmetric models can be a candidate for cold dark matter;

The minimal supersymmetric standard model (MSSM) [30] is the minimal extension of the standard model (SM) with (i) a supersymmetric partner for each SM particle and every Higgs boson, (ii) two Higgs doublets $H_{1}$ and $H_{2}$ such that $H_{1}$ couples to the fermions with $t_{3}=-1 / 2$ and $H_{2}$ couples to the fermions with $t_{3}=+1 / 2$, (iii) a Higgs mixing parameter $\mu$ assumed to be real, (iv) three real symmetric $3 \times 3$ matrices of squark mass-squared parameters, $M_{\tilde{Q}}^{2}, M_{\tilde{U}}^{2}$ and $M_{\tilde{D}}^{2}$, (v) two real symmetric $3 \times 3$ matrices of slepton mass-squared parameters, $M_{\tilde{L}}^{2}$ and $M_{\tilde{E}}^{2}$, (vi) three real $3 \times 3$ matrices of trilinear Yukawa parameters, $A_{U}, A_{D}$ and $A_{E}$, and (vii) a conserved $R$-parity $R \equiv(-1)^{3 B+L+2 S}=(-1)^{3(B-L)+2 S}$. In the MSSM, the lightest supersymmetric particle (LSP) is stable and is a very attractive candidate for cosmological dark matter.

The neutral electroweak gauginos $\left(\tilde{B}\right.$ and $\left.\tilde{W}^{3}\right)$ and the Higgsinos $\left(\tilde{H}_{1}^{0}\right.$ and $\left.\tilde{H}_{2}^{0}\right)$ have the same quantum numbers. They mix and generate four mass eigenstates called neutralinos. The neutralino mass matrix in the basis of $\left(\tilde{B}, \tilde{W}^{3}, \tilde{H}_{1}^{0}, \tilde{H}_{2}^{0}\right)$ states is

$$
\mathcal{M}_{N}=\left(\begin{array}{cccc}
M_{1} & 0 & -M_{Z} \cos \beta \sin \theta_{W} & M_{Z} \sin \beta \sin \theta_{W} \\
0 & M_{2} & M_{Z} \cos \beta \cos \theta_{W} & -M_{Z} \sin \beta \cos \theta_{W} \\
-M_{Z} \cos \beta \sin \theta_{W} & M_{Z} \cos \beta \cos \theta_{W} & 0 & -\mu \\
M_{Z} \sin \beta \sin \theta_{W} & -M_{Z} \sin \beta \cos \theta_{W} & -\mu & 0
\end{array}\right) .
$$

This mass matrix is symmetric and can be diagonalized by a single matrix [30]. The diagonalization is given by the following analytic expressions [52,53.

$$
\begin{aligned}
& \epsilon_{1} M_{\chi_{1}^{0}}=-\left(\frac{1}{2} a-\frac{1}{6} C_{2}\right)^{1 / 2}+\left[-\frac{1}{2} a-\frac{1}{3} C_{2}+\frac{C_{3}}{\left(8 a-\frac{8}{3} C_{2}\right)^{1 / 2}}\right]^{1 / 2}+\frac{1}{4}\left(M_{1}+M_{2}\right) \\
& \epsilon_{2} M_{\chi_{2}^{0}}=+\left(\frac{1}{2} a-\frac{1}{6} C_{2}\right)^{1 / 2}-\left[-\frac{1}{2} a-\frac{1}{3} C_{2}-\frac{C_{3}}{\left(8 a-\frac{8}{3} C_{2}\right)^{1 / 2}}\right]^{1 / 2}+\frac{1}{4}\left(M_{1}+M_{2}\right) \\
& \epsilon_{3} M_{\chi_{3}^{0}}=-\left(\frac{1}{2} a-\frac{1}{6} C_{2}\right)^{1 / 2}-\left[-\frac{1}{2} a-\frac{1}{3} C_{2}+\frac{C_{3}}{\left(8 a-\frac{8}{3} C_{2}\right)^{1 / 2}}\right]^{1 / 2}+\frac{1}{4}\left(M_{1}+M_{2}\right) \\
& \epsilon_{4} M_{\chi_{4}^{0}}=+\left(\frac{1}{2} a-\frac{1}{6} C_{2}\right)^{1 / 2}+\left[-\frac{1}{2} a-\frac{1}{3} C_{2}-\frac{C_{3}}{\left(8 a-\frac{8}{3} C_{2}\right)^{1 / 2}}\right]^{1 / 2}+\frac{1}{4}\left(M_{1}+M_{2}\right)
\end{aligned}
$$

where $\epsilon_{i}$ is the sign of the $i$ th eigenvalue of the neutralino mass matrix, and

\footnotetext{
${ }^{3}$ The $R$-parity is defined as $R=(-1)^{3 B+L+2 S}$, where $B=$ Baryon number, $L=$ Lepton number and $S=$ Spin. $R=+1$ for the SM particles and Higgs bosons, and $R=-1$ for their superpartners.
} 


$$
\begin{aligned}
C_{2}= & \left(M_{1} M_{2}-M_{Z}^{2}-\mu^{2}\right)-\frac{3}{8}\left(M_{1}+M_{2}\right)^{2} \\
C_{3}= & -\frac{1}{8}\left(M_{1}+M_{2}\right)^{3}+\frac{1}{2}\left(M_{1}+M_{2}\right)\left(M_{1} M_{2}-M_{Z}^{2}-\mu^{2}\right)+\left(M_{1}+M_{2}\right) \mu^{2} \\
& +\left(M_{1} \cos ^{2} \theta_{W}+M_{2} \sin ^{2} \theta_{W}\right) M_{Z}^{2}-\mu M_{Z}^{2} \sin 2 \beta \\
C_{4}= & \left(M_{1} \cos ^{2} \theta_{W}+M_{2} \sin ^{2} \theta_{W}\right) M_{Z}^{2} \mu \sin 2 \beta-M_{1} M_{2} \mu^{2} \\
& +\frac{1}{4}\left(M_{1}+M_{2}\right)\left[\left(M_{1}+M_{2}\right) \mu^{2}+\left(M_{1} \cos ^{2} \theta_{W}+M_{2} \sin ^{2} \theta_{W}\right) M_{Z}^{2}-\mu M_{Z}^{2} \sin 2 \beta\right] \\
& +\frac{1}{16}\left(M_{1} M_{2}-M_{Z}^{2}-\mu^{2}\right)\left(M_{1}+M_{2}\right)^{2}-\frac{3}{256}\left(M_{1}+M_{2}\right)^{4}, \\
a= & \frac{1}{2^{1 / 3}} \operatorname{Re}\left[-S+i(D / 27)^{1 / 2}\right]^{1 / 3}, \\
D= & -4 U^{3}-27 S^{2}, \quad U=-\frac{1}{3} C_{2}^{2}-4 C_{4}, \quad S=-C_{3}^{2}-\frac{2}{27} C_{2}^{3}+\frac{8}{3} C_{2} C_{4} .
\end{aligned}
$$

The masses given by the above expressions are usually not in the order $M_{\chi_{1}^{0}}<M_{\chi_{2}^{0}}<M_{\chi_{3}^{0}}<$ $M_{\chi_{4}^{0}}$, but the eigenstates can be relabeled.

The mixing matrix $Z$, defined by $\mathcal{M}_{\text {diag }}=Z \mathcal{M}_{N} Z^{-1}$, is given by analytic expressions 52,53

$$
\begin{aligned}
\frac{z_{i 2}}{z_{i 1}} & =-\frac{1}{\tan \theta_{W}} \frac{M_{1}-\epsilon_{i} M_{\chi_{i}^{0}}}{M_{2}-\epsilon_{i} M_{\chi_{i}^{0}}}, \\
\frac{z_{i 3}}{z_{i 1}} & =\frac{\mu\left[M_{2}-\epsilon_{i} M_{\chi_{i}^{0}}\right]\left[M_{1}-\epsilon_{i} M_{\chi_{i}^{0}}\right]-M_{Z}^{2} \sin \beta \cos \beta\left[\left(M_{1}-M_{2}\right) \cos ^{2} \theta_{W}+M_{2}-\epsilon_{i} M_{\chi_{i}^{0}}\right]}{M_{Z}\left[M_{2}-\epsilon_{i} M_{\chi_{i}^{0}}\right] \sin \theta_{W}\left[\mu \cos \beta+\epsilon_{i} M_{\chi_{i}^{0}} \sin \beta\right]}, \\
\frac{z_{i 4}}{z_{i 1}} & =\frac{-\epsilon_{i} M_{\chi_{i}^{0}}\left[M_{2}-\epsilon_{i} M_{\chi_{i}^{0}}\right]\left[M_{1}-\epsilon_{i} M_{\chi_{i}^{0}}\right]-M_{Z}^{2} \cos ^{2} \beta\left[\left(M_{1}-M_{2}\right) \cos ^{2} \theta_{W}+M_{2}-\epsilon_{i} M_{\chi_{i}^{0}}\right]}{M_{Z}\left[M_{2}-\epsilon_{i} M_{\chi_{i}^{0}}\right] \sin \theta_{W}\left[\mu \cos \beta+\epsilon_{i} M_{\chi_{i}^{0}} \sin \beta\right]},
\end{aligned}
$$

and

$$
z_{i 1}=\left[1+\left(\frac{z_{i 2}}{z_{i 1}}\right)^{2}+\left(\frac{z_{i 3}}{z_{i 1}}\right)^{2}+\left(\frac{z_{i 4}}{z_{i 1}}\right)^{2}\right]^{-1 / 2} .
$$

In most SUSY models, the lightest neutralino $\left(\chi_{1}^{0}\right)$ is the preferred the dark matter particle; it is a mixture of gauginos and higgsinos,

$$
\chi_{1}^{0}=z_{11} \tilde{B}+z_{12} \tilde{W}^{3}+z_{13} \tilde{H}_{1}^{0}+z_{14} \tilde{H}_{2}^{0} .
$$

The gaugino fraction of $\chi_{1}^{0}$ can be defined as

$$
f_{G}=z_{11}^{2}+z_{12}^{2}
$$

and its higgisino fraction as

$$
f_{H}=z_{13}^{2}+z_{14}^{2}
$$


The lightest neutralino is more gaugino-like for $\mu>M_{2}$ and gauginos annihilate mostly into heavy quarks; the lightest neutralino becomes more higgisino-like for $\mu \lesssim M_{2}$ and the higgsinos annihilate dominantly into gauge bosons.

The minimal supersymmetric model has 63 free parameters with real mass matrices and couplings. To make the analysis of the MSSM tractable, we assume a common value for the masses of scalar fermions and the trilinear couplings $\left(M_{\mathrm{SUSY}}=m_{\tilde{f}}=A_{f}\right)$. The most relevant of the remaining free parameters are the $\mathrm{SU}(2)$ gaugino mass $\left(M_{2}\right)$, the Higgs mixing parameter $(\mu)$, the ratio of vacuum expectation values (VEVs) of Higgs fields $\left(\tan \beta \equiv v_{2} / v_{1}\right)$ and the CP-odd Higgs-boson mass $\left(m_{A}\right)$.

\section{A. The Effects of Muon Energy Threshold}

The indirect search for SUSY neutralino dark matter with high energy neutrinos is a promising approach to detect the annihilations of neutralino dark matter accumulated in the Sun [31]- [47]. To obtain realistic rate estimates, we consider the muon detection threshold, quark hadronization, and solar absorption.

For neutrinos from $\chi \bar{\chi} \rightarrow W^{+} W^{-} \rightarrow \mu^{+} \nu_{\mu}+X$ the average energy of the muon is about $m_{\chi} / 4$; it is about $m_{\chi} / 6$ for neutrinos from $\chi \bar{\chi} \rightarrow Q \bar{Q} \rightarrow \mu^{+} \nu_{\mu} q+X$. The detection energy threshold for muons makes a significant impact on the indirect detection rate. The effect of muon threshold on the indirect detection rate from MSSM neutralino dark matter annihilations in the Sun is demonstrated in Figs. 1, 2 and 3 for $m_{\chi_{1}^{0}}=200 \mathrm{GeV}, 500 \mathrm{GeV}$, and $1000 \mathrm{GeV}$, with two values of $\tan \beta$ and two sets of $M_{2}$ and $\mu$ : (i) $\mu \gtrsim M_{2}$ such that the lightest neutralino is more gaugino-like, and (ii) $\mu<M_{2}$ such that the $\chi_{1}^{0}$ is more higgsinolike. For simplicity, $M_{\mathrm{SUSY}}=1.5 m_{\chi_{1}^{0}}$ is assumed for a common squark mass $\left(m_{\tilde{q}}\right)$, a common slepton mass $\left(m_{\tilde{\ell}}\right)$, and a common trilinear coupling $\left(A_{u}=A_{d}=A_{\ell}\right)$. We note that the higgsino-like neutralino has a substantially higher rate with a higher average muon energy than a gaugino-like neutralino. In addition, a higher detector energy threshold effectively reduces the indirect detection rate for background and the signal with $m_{\chi_{1}^{0}} \lesssim 200 \mathrm{GeV}$. For $m_{\chi_{1}^{0}} \gtrsim 500 \mathrm{GeV}$, the indirect detection rate is not very sensitive to the muon energy threshold.

\section{B. Hadronization of Quarks and Solar Absorption}

There are uncertainties in the signal rate calculation form hadronization of quarks and from solar absorption that we now discuss. For a gaugino-like neutralino, the dominant annihilation modes are $b \bar{b}$ or $\tau \bar{\tau}$ for $m_{\chi}<m_{W^{ \pm}}$and $b \bar{b}$ for $m_{\chi}>m_{W^{ \pm}}$. For a higgsino-like neutralino, the dominant modes are $Z Z$ or $W^{+} W^{-}$for $m_{\chi}<m_{t}$ and $t \bar{t}$ for $m_{\chi}>m_{t}$ [54]. The effects of hadronization of heavy quarks into heavy mesons or heavy baryons reduces the energy of the neutrinos from the semileptonic decays [54].

Moreover, neutrinos produced in the Sun may be absorbed before they escape the solar medium. We use the Ritz and Seckel parameterization up to energies around $1 \mathrm{TeV}$ for all neutrino producing annihilation modes [36] to estimate the neutrino absorption losses. Above the $\mathrm{TeV}$ scale, extra corrections are treated in a manner similar to work by Edsjo [55. 
Indirect detectors generally have neutrino energy thresholds of at least a few tens of GeV. We have chosen $25 \mathrm{GeV}$ for the muon energy threshold in our analysis which corresponds to a neutrino energy of about $50 \mathrm{GeV}$.

The combined effects of hadronization, solar absorption and muon threshold energy can vary from total obliteration of the neutrino signal for a neutralino mass around $100 \mathrm{GeV}$ to merely a reduction by less than one half in neutrino signal for a neutralino mass close to $1 \mathrm{TeV}$. Figures 1, 2 and 3 show representative calculations for a typical MSSM neutralino dark matter with a mass of $200 \mathrm{GeV}, 500 \mathrm{GeV}$ and $1000 \mathrm{GeV}$, respectively. The effects become far less significant for heavier neutralinos. However, higher energy neutrinos have a larger solar absorption rate, therefore, slightly more neutrinos from annihilations of 1000 $\mathrm{GeV}$ neutralinos are absorbed than those from $500 \mathrm{GeV}$ neutralinos.

\section{Relic Density and Indirect Detection Rate}

For large $\tan \beta$ with heavy SUSY particles, the neutralino annihilation cross section is enhanced by the s-channel diagrams involving broad resonance poles of Higgs bosons, $\chi_{1}^{0} \chi_{1}^{0} \rightarrow A^{0}, H^{0} \rightarrow b \bar{b}, \tau \bar{\tau}$, where $A^{0}$ is the $\mathrm{CP}$ odd pseudoscalar $(A)$, and $H^{0}$ is the heavier $\mathrm{CP}$ even scalar $(H)$. Consequently, the neutralino relic density lies within the cosmologically interesting region in much of the parameter space in SUSY models [58 63].

In Figure 4, we present relic density of the neutralino dark matter $\left(\Omega_{\chi_{1}^{0}} h^{2}\right)$ versus $M_{2}$ for several values of $\mu$ with $M_{\text {SUSY }}$ taken to be the larger of $300 \mathrm{GeV}$ and $1.5 m_{\chi_{1}^{0}}$. For 200 $\mathrm{GeV} \lesssim M_{2} \lesssim 1200 \mathrm{GeV}$ with $3 \lesssim \tan \beta \lesssim 50$ and $M_{\text {SUSY }} \simeq \max \left(300 \mathrm{GeV}, 1.5 \mathrm{~m}_{\chi_{1}^{0}}\right)$. the relic density can be in the cosmological interesting region.

The indirect detection rate $\left(d N_{\text {ID }} / d A\right)$ in events $/ \mathrm{km}^{2} /$ year is presented versus $M_{2}$ in Fig. 5. Here we have assumed a detector threshold energy of muons to be $25 \mathrm{GeV}$. Figure 6 shows regions in the parameter plane of $\left(M_{2}, \mu\right)$ that may yield an indirect detection rate in events/ $\mathrm{km}^{2} /$ year: (i) $d N_{\text {ID }}>100$ (dark shading), (ii) $100>d N_{\text {ID }}>10$ (intermediate) (iii) $d N_{\text {ID }}<10$ (light shading). Parameters in the blank regions do not generate a cosmologically interesting relic density $\left(0.05 \leq \Omega_{\chi_{1}^{0}} \leq 0.3\right)$ for the neutralino dark matter.

The indirect detection rate can be significant ( $>10$ events $/ \mathrm{km}^{2} /$ year) in large regions of the parameter space with $10 \lesssim \tan \beta \lesssim 50$ : (i) in the neighborhood of $M_{2} \sim 500 \mathrm{GeV}$ and $\mu \sim 500 \mathrm{GeV}$, (ii) in the neighborhood of $M_{2} \sim 4000 \mathrm{GeV}$ and $\mu \sim 1200 \mathrm{GeV}$, (iii) in a narrow band with $M_{2} \gtrsim 1200 \mathrm{GeV}$ and $M_{2} \sim 2 \mu$, and (iv) in a narrow band with $M_{2} \sim 400$ $\mathrm{GeV}$ and $\mu \gtrsim 1200 \mathrm{GeV}$ for $\tan \beta \sim 50$.

\section{THE MINIMAL SUPERGRAVITY MODEL}

In the minimal supergravity (mSUGRA) model it is assumed that SUSY is broken in a hidden sector with SUSY breaking communicated to the observable sector through gravitational interactions, leading naturally to a common scalar mass $\left(m_{0}\right)$, a common gaugino mass $\left(m_{1 / 2}\right)$, a common trilinear coupling $\left(A_{0}\right)$, and a bilinear coupling $\left(B_{0}\right)$, at the GUT scale $\left(M_{\mathrm{GUT}} \sim 2 \times 10^{16} \mathrm{GeV}\right)$. Through minimization of the Higgs potential, the $B$ parame- 
ter and magnitude of the superpotential Higgs mixing parameter $\mu$ are related to $\tan \beta$ and $M_{Z}$.

The mass matrix of the charginos in the basis of the weak eigenstates $\left(\tilde{W}^{ \pm}, \tilde{H}^{ \pm}\right)$has the following form

$$
M_{C}=\left(\begin{array}{cc}
M_{2} & \sqrt{2} M_{W} \sin \beta \\
\sqrt{2} M_{W} \cos \beta & \mu
\end{array}\right) .
$$

The sign of the $\mu$ term in Eq. (30) establishes our sign convention. Analyses of $b \rightarrow s \gamma$ decay 64, 68, 66 69, 69 and muon g-2 measurements [70] - 89] strongly favor $\mu>0$. We shall only consider $\mu>0$ and $A_{0}=0$.

The SUSY particle masses and couplings at $M_{Z}$ can be predicted by the evolution of renormalization group equations (RGEs) from $M_{\mathrm{GUT}}$. The gaugino masses at the weak scale $\left(M_{Z}\right)$ have the following relations:

- $M_{1} / \alpha_{1}=M_{2} / \alpha_{2}=M_{3} / \alpha_{3}$,

- $M_{1} \simeq 0.4 m_{1 / 2}, \quad M_{2} \simeq 0.8 m_{1 / 2}, \quad M_{3} \simeq 2.7 m_{1 / 2}$

- $m_{\chi_{1}^{0}}: m_{\chi_{2}^{0}}: m_{\chi_{1}^{ \pm}}: m_{\tilde{g}} \simeq 1: 2: 2: 6$.

We calculate SUSY particle masses at $M_{Z}$ and the masses and couplings in the Higgs sector with one loop corrections in the one-loop effective potential at the scale $Q=\sqrt{m_{\tilde{t}_{L}} m_{\tilde{t}_{R}}}$ [57.61. At the scale $Q$, the RGE improved one-loop corrections approximately reproduce the dominant two loop corrections 90 95] to the mass of the lighter CP-even scalar.

We impose the following theoretical requirements on the RGE evolution:

(i) radiative electroweak symmetry breaking (EWSB) is achieved,

(ii) the correct vacuum for EWSB is obtained (tachyon free), and

(iii) the lightest SUSY particle is the lightest neutralino.

In Figure 7, we present the relic density of the mSUGRA neutralino dark matter $\left(\Omega_{\chi_{1}^{0}} h^{2}\right)$ versus $m_{1 / 2}$ for several values of $m_{0}$. For $\tan \beta \lesssim 35$, cosmologically interesting relic densities are found for $m_{1 / 2} \lesssim 500 \mathrm{GeV}$. For $\tan \beta \lesssim 50$, dips in $\Omega_{\chi_{1}^{0}}$ appear near resonance poles at $2 m_{\chi_{1}^{0}} \simeq m_{H}, m_{A}$.

Figure 8 shows the higgsino fraction of the lightest neutralino in the mSUGRA model. In most the of the $m_{1 / 2}, m_{0}$ plane, $\chi_{1}^{0}$ is mostly gaugino-like even with $m_{0}>1000 \mathrm{GeV}$ 96,97. Only in a narrow band when values of $m_{1 / 2}$ and $m_{0}$ are close to theoretically excluded region, can the lightest neutralino have a higgsino fraction larger than $10 \%$. The region excluded by the $m_{\chi_{1}^{+}} \lesssim 103 \mathrm{GeV}$ limit from the chargino search [98] at LEP2 is indicated.

The indirect detection rate $\left(d N_{\mathrm{ID}} / d A\right)$ in events $/ \mathrm{km}^{2} /$ year versus $m_{1 / 2}$ is presented in Fig. 9 for the mSUGRA model. Again we take the detector threshold energy of the muon to be $25 \mathrm{GeV}$. Figure 10 shows regions in the parameter plane of $\left(m_{1 / 2}, m_{0}\right)$ that may yield an indirect detection rate in events $/ \mathrm{km}^{2} /$ year with: (i) $10>d N_{\mathrm{ID}} / d A>1$ (intermediateshading) and (ii) $d N_{\mathrm{ID}} / d A<1$ (light shading). There is no region that has $d N_{\mathrm{ID}} / d A>10$. Parameters in the blank regions do not generate a cosmologically interesting relic density $\left(0.05 \leq \Omega_{\chi_{1}^{0}} \leq 0.3\right)$ for the neutralino dark matter. For $\tan \beta=50$, the relic density is suppressed below the cosmologically interesting value when $2 m_{\chi_{1}^{0}} \simeq m_{H}, m_{A}$. That leads to a blank narrow band in Fig. 10(b). 


\section{SUPERGRAVITY MODELS WITH NONUNIVERSAL HIGGS-BOSON MASSES AT THE GUT SCALE}

We next consider a supergravity (SUGRA) unified model with non-universal boundary conditions for the Higgs bosons at the unified scale $\left(m_{\mathrm{GUT}}\right)$. We parameterize the GUT-scale Higgs masses ast

$$
m_{H_{i}}(\mathrm{GUT})=\left(1+\delta_{i}\right) m_{0}=\rho_{i} m_{0}, i=1,2
$$

where $\delta_{i}=-1$ and 2 correspond to $\rho_{i}=0$ and 2 The universal mSUGRA model has $\delta_{1}=\delta_{2}=0$, while $\delta_{i}=-1$ and $\delta_{i}=2$ correspond to $m_{H_{i}}(\mathrm{GUT})=0$ and $m_{H_{i}}(\mathrm{GUT})=2 m_{0}$. The nonuniversality of Higgs-boson masses at $m_{\mathrm{GUT}}$ can significantly affect the values of Higgs masses and couplings at the weak scale.

In Figures 11 and 12 we present the relic density of neutralino dark matter $\left(\Omega_{\chi_{1}^{0}} h^{2}\right)$ versus $\delta_{1}$ and $\delta_{2}$ for several values of $m_{0}$. For $\tan \beta \lesssim 10$, the non-universality of the Higgs masses makes slight only impact on relic density of the neutralino dark matter. For $\tan \beta \gtrsim 35$, non-universality affects the weak-scale Higgs-bosons masses, the value of the $\mu$ parameter and the neutralino relic density. For $\tan \beta \sim 35$ and 50, dips appear when $2 m_{\chi_{1}^{0}}$ becomes close to $m_{H}, m_{A}$.

In Fig. 13, we present the higgsino fraction of the lightest neutralino $\left(f_{H}\right)$ as defined in Eq. (29) in the plane of $\left(\delta_{1}, \delta_{2}\right)$ with $m_{1 / 2}=m_{0}=400 \mathrm{GeV}, A_{0}=0$. For $\tan \beta \sim 3$, an increase in $\delta_{1}$ or $\delta_{2}$ generates a larger Higgsino fraction for $\chi_{1}^{0}$, while for $\tan \beta \gtrsim 10$, a decrease in $\delta_{1}$ or an increase in $\delta_{2}$ can make $f_{H}$ larger for $\chi_{1}^{0}$. The higgsino fraction of $\chi_{1}^{0}$ is very sensitive to the value of $\delta_{2}$.

The indirect detection rate $\left(d N_{\mathrm{ID}} / d A\right)$ in events $/ \mathrm{km}^{2} /$ year versus $m_{1 / 2}$ is presented in Figs. 14 and 15. The threshold detection energy of the muon is assumed be $25 \mathrm{GeV}$. For $\tan \beta \gtrsim 35$, the indirect detection rate can be large for chosen values of the parameters. Figures 16, 17 and 18 show regions in the parameter plane of $\left(m_{1 / 2}, m_{0}\right)$ that may yield an indirect detection rate in events $/ \mathrm{km}^{2} /$ year, (i) $d N_{\text {ID }}>10$ (dark shading), (ii) $10>d N_{\text {ID }}>1$ (intermediate shading) (iii) $d N_{\mathrm{ID}}<1$ (light shading). Three cases are considered: (a) $\delta_{1}=-0.5$ and $\delta_{2}=0$ (Fig. 16), (b) $\delta_{1}=0$ and $\delta_{2}=0.5$ (Fig. 17), and (a) $\delta_{1}=-0.5$ and $\delta_{2}=0.5$ (Fig. 18). Parameters in the blank regions do not generate a cosmologically interesting relic density $\left(0.05 \leq \Omega_{\chi_{1}^{0}} \leq 0.3\right)$ for the neutralino dark matter. For $\tan \beta \sim 50$, non-universality can significantly enhance the indirect detection rate for the neutralino dark matter.

For $\tan \beta \sim 10$, the predicted indirect detection rate is interesting only in a very small band near the theoretically excluded region with $m_{1 / 2} \gtrsim 600 \mathrm{GeV}$ and $m_{0} \sim 3 m_{1 / 2}$. For $\tan \beta \sim 50$, the indirect detection rate is significant in a large region of parameter space with $m_{1 / 2} \lesssim 1000 \mathrm{GeV}$ and $m_{0} \lesssim 800 \mathrm{GeV}$.

\footnotetext{
${ }^{4}$ In Refs. 100,101, $m_{H_{i}}^{2}(\mathrm{GUT})$ are parameterized to be $\left(1+\delta_{i}\right) m_{0}^{2}$.
} 


\section{PROSPECTS FOR INDIRECT DETECTION}

For a very large volume second generation neutrino detector with superb angular resolution and large statistics for atmospheric neutrinos of order $10^{4}$ events per year for IceCube, this background can be reduced, in fact, essentially eliminated. Because the atmopheric neutrino background will be known with high precision, the number of events in the angular bin of the Sun can be determined by interpolation from adjacent bins. It should be possible to estimate the rate of atmospheric neutrinos to the level of a few events, and to achieve a background much smaller than the fluctuations on the events in the bin. In such a detector, the dominant background comes from neutrinos produced by cosmic ray interacting in the Sun's corona. This background is between 10 and 5 events per year per square kilometer for muon thresholds between 25 and $100 \mathrm{GeV}$ [43].

The next generation high energy neutrino telescopes such as IceCube and ANTARES with effective area on the order of $1 \mathrm{~km}^{2}$ may have the capability to make the first detections of supersymmetric cold dark matter through the detection of neutrinos produced via annihilation of heavy neutralino in the Sun. Considering muon thresholds ranging from $25 \mathrm{GeV}$ to $100 \mathrm{GeV}$ (reflecting a variety of detectors and their variation in design), our calculations show that a significant fraction of the MSSM parameter space with $\tan \beta \gtrsim 10$ should produce a muon event rate above backgrounds in such detectors. The prospects for searching mSUGRA parameter space are less optimistic than in the MSSM. If non-universal boundary conditions are considered, the prospects of detecting SUGRA neutralino dark matter with such detectors are increased.

We have focused on the flux of muons $\left(\phi_{\mu}\right)$ generated by high-energy neutrinos (in units of 'events $/ \mathrm{km}^{2} /$ year'). The event rate for a thin detector such as the AMANDA can be obtained from $\phi_{\mu} \times A_{\text {eff }}$, where $A_{\text {eff }}$ is the effective area. For detectors of a volume $\gtrsim 1 \mathrm{~km}^{3}$

such as the IceCube, the thin detector approximation is valid for muon energies larger than a few hundred GeV. For lower energy muons, the event rates will be higher than those obtained from thin detector approximation [43].

The IceCube detector will have good angular and energy resolution for high energy muons [18]. The measurement of muon angular distribution can be used to focus the search window around the direction from the Sun and to reduce the background effectively. The atmospheric neutrino background in a solar pixel will be determined accurately. The significance of the neutralino signal at the IceCube will be greatly enhanced with suitable background subtraction.

The question of naturalness and fine tuning arises for models with heavy neutralinos. Recently, it has been shown that $\mathrm{TeV}$ scale values of $m_{0}$ can be natural [99]. The purpose of this paper does not lie with this issue and we put forward our results only as a scan of the described parameter space setting aside the subjective criterion of naturalness requirements. However, because of naturalness, we do not consider the super-heavy models in which the lightest neutralino has mass of order several TeV or greater.

\section{CONCLUSIONS}

High energy muons produced by neutrinos from relic neutralino annihilation in the Sun can lead to promising signals in the ice and the water detectors of high energy neutrinos. 
We find that:

(i) The effects of detector threshold, hadronization and solar absorption can be essential in the evaluation of indirect detection rates, especially for neutralinos with mass below a few hundred $\mathrm{GeV}$.

(ii) In large portions of the MSSM parameter space with $\tan \beta \gtrsim 10$, the indirect detection rate is predicted to be greater than ten events $/ \mathrm{km}^{2} /$ year. However, the indirect search for neutralino annihilation may be difficult for $\tan \beta \lesssim 10$ or $m_{\chi} \lesssim 200 \mathrm{GeV}$.

(iii) The indirect search for the mSUGRA neutralino dark matter will be challenging. Only several years of observation with a square kilometer detector will likely lead to a discovery. SUGRA models with non-universal boundary conditions and $\tan \beta \gtrsim 35$ give more interesting rates.

(iv) For large values of large $\tan \beta$ the neutralino annihilation cross section and the indirect detection rate are enhanced by the s-channel diagrams involving Higgs bosons, $\chi_{1}^{0} \chi_{1}^{0} \rightarrow$ $A^{0}, H^{0} \rightarrow b \bar{b}, \tau \bar{\tau}$, where $A^{0}$ is the CP odd pseudoscalar, and $H^{0}$ is the heavier CP even scalar, and high energy neutrinos are produced in the $b$ and $\tau$ decays.

(v) For the AMANDA experiment, with a muon energy threshold of $25 \mathrm{GeV}$ the background muon flux from atmospheric neutrinos in a pixel containing the neutralino signal is about 4.6 events $/\left(10^{4} \mathrm{~m}^{2}\right) /$ year. This background flux is larger than the signal rate in most regions of the parameter space in SUSY models. To improve the significance of signal over background, a higher muon energy threshold will be advantageous if $m_{\chi} \gtrsim 500 \mathrm{GeV}$.

(vi) For the IceCube and the ANTARES experiments, the large number of background events will make background subtraction possible with only a small remaining irreducible background 43] and allow measurement of the neutralino annihilation signal.

(vii) SUSY neutralino dark matter with a mass larger than about $200 \mathrm{GeV}$ offers great promise for indirect detection experiments. Together with direct detection experiments of neutralino dark matter and accelerator experiments at the upgraded Tevatron and the CERN Large Hadron Collider, high-energy neutrino telescopes will be able to survey large regions of parameter space beyond present experiments.

\section{ACKNOWLEDGMENTS}

C.K. thanks Tzi-Hong Chieuh and George Hou for hospitality at the National Taiwan University where part of the research was completed. This research was supported in part by the U.S. Department of Energy under Grants No. DE-FG02-95ER40896 and No. DEFG03-98ER41066, and in part by the University of Wisconsin Research Committee with funds granted by the Wisconsin Alumni Research Foundation. 


\section{REFERENCES}

[1] R. Tripp and D. Branch, Astrophys. J. 525, 209 (1999).

[2] W. L. Freedman, Phys. Rept. 333, 13 (2000).

[3] J. R. Mould et al., Astrophys. J. 529, 786 (2000).

[4] K.G. Begeman, A.H. Broeils, R.H. Sanders, Mon. Not. R. Astr. Soc. 249, 523 (1991).

[5] J.J. Mohr, B. Mathiesen and A.E. Evrard, Astrophys. J. 517, 627 (1999).

[6] S. Perlmutter et al. [Supernova Cosmology Project Collaboration], Astrophys. J. 517, 565 (1999).

[7] The BOOMERANG collaboration: P. de Bernardis et al., Nature 404, 955 (2000); A. E. Lange et al., Phys. Rev. D 63, 042001 (2001); C. B. Netterfield et al., astro$\mathrm{ph} / 0104460$.

[8] The MAXIMA collaboration: S. Hanany et al., Astrophys. J. Lett. 545, 5 (2000); A. Balbi et al., Astrophys. J. 545, L1 (2000).

[9] The DASI collaboration: C. Pryke et al., astro-ph/0104490.

[10] S. Burles, K. M. Nollett and M. S. Turner, Phys. Rev. Lett. 82, 4176 (1999); Phys. Rev. D 63, 063512 (2001).

[11] M. Fukugita, C. J. Hogan and P. J. Peebles, Astrophys. J. 503, 518 (1998).

[12] M. Davis, G. Efstathiou, C. S. Frenk and S. D. White, Astrophys. J. 292, 371 (1985).

[13] G. Steigman, Ann. Rev. Nucl. Part. Sci. 29, 313 (1979); J.R. Primack, D. Seckel and B. Sadoulet, Ann. Rev. Nucl. Part. Sci. 38, 751 (1988).

[14] H. Goldberg, Phys. Rev. Lett. 50, 1419 (1983).

[15] J. Ellis, J. S. Hagelin, D. V. Nanopoulos, K. Olive and M. Srednicki, Nucl. Phys. B 238, 453 (1984).

[16] G. Jungman, M. Kamionkowski and K. Griest, Phys. Rept. 267, 195 (1996); http://t8web.lanl.gov/people/jungman/neut-package.html. We have used the Neutdriver package to calculate the indirect detection rate in the MSSM.

[17] The AMANDA Collaboration, E. Andres et al., Nature 410, 441 (2001).

[18] The IceCube, http://pheno.physics.wisc.edu/icecube/.

[19] The ANTARES Collaboration, E. Aslanides et al., astro-ph/9907432 (1999).

[20] E. I. Gates, G. Gyuk and M. S. Turner, Astrophys. J. 449, L123 (1995) astroph/9505039.

[21] M. Kamionkowski and A. Kinkhabwala, Phys. Rev. D 57, 3256 (1998) hepph/9710337.

[22] G. R. Knapp et.al., Astron. J. 83, 1585 (1978).

[23] F. J. Kerr and D. Lynden-Bell, Mon. Not. R. Astr. Soc. 221, 1023 (1986).

[24] J. A. R. Caldwell and J. M. Coulsen, Astron. J. 93, 1090 (1987).

[25] F. Halzen, presented at International Symposium on Particle Theory and Phenomenology, Ames, Iowa, 22-24 May 1995, University of Wisconsin Report MADPH-95-902 (1995) astro-ph/9508020.

[26] T. K. Gaisser, F. Halzen and T. Stanev, Phys. Rept. 258, 173 (1995) hep-ph/9410384.

[27] P. Lipari and T. Stanev, Phys. Rev. D 44, 3543 (1991).

[28] T. K. Gaisser and T. Stanev, Phys. Rev. D 31, 2770 (1985).

[29] T. K. Gaisser and A. F. Grillo, Phys. Rev. D 36, 2752 (1987).

[30] H.P. Nilles, Phys. Rep. 110, 1 (1984); H. Haber and G. Kane, Phys. Rep. 117, 75 
(1985); X. Tata, Lectures presented at the IX Jorge Swieca Summer School, Campos do Jordão, Brazil, February 1997, UH-511-872-97, hep-ph/9706307.

[31] J. Silk, K. Olive and M. Srednicki, Phys. Rev. Lett. 55, 257 (1985).

[32] K. Freese, Phys. Lett. B 167, 295 (1986).

[33] L. M. Krauss, M. Srednicki and F. Wilczek, Phys. Rev. D 33, 2079 (1986).

[34] T. K. Gaisser, G. Steigman and S. Tilav, Phys. Rev. D 34, 2206 (1986).

[35] J. S. Hagelin, K. W. Ng and K. A. Olive, Phys. Lett. B 180, 375 (1986); K. Ng, K. A. Olive and M. Srednicki, Phys. Lett. B 188, 138 (1987).

[36] S. Ritz and D. Seckel, Nucl. Phys. B 304, 877 (1988).

[37] G. F. Giudice and E. Roulet, Nucl. Phys. B 316, 429 (1989).

[38] M. Kamionkowski, Phys. Rev. D 44, 3021 (1991).

[39] F. Halzen, T. Stelzer and M. Kamionkowski, Phys. Rev. D 45, 4439 (1992).

[40] R. Gandhi, J. L. Lopez, D. V. Nanopoulos, K. Yuan and A. Zichichi, Phys. Rev. D 49, 3691 (1994)

[41] E. Diehl, G. L. Kane, C. Kolda and J. D. Wells, Phys. Rev. D 52, 4223 (1995).

[42] V. Berezinsky, A. Bottino, J. Ellis, N. Fornengo, G. Mignola and S. Scopel, Astropart. Phys. 5, 333 (1996).

[43] L. Bergstrom, J. Edsjo and P. Gondolo, Phys. Rev. D 55, 1765 (1997); Phys. Rev. D 58, 103519 (1998).

[44] L. Bergstrom, J. Edsjo and M. Kamionkowski, Astropart. Phys. 7, 147 (1997).

[45] A. Corsetti and P. Nath, Int. J. Mod. Phys. A 15, 905 (2000).

[46] A. E. Faraggi, K. A. Olive and M. Pospelov, Astropart. Phys. 13, 31 (2000).

[47] J. L. Feng, K. T. Matchev and F. Wilczek, Phys. Rev. D 63, 045024 (2001).

[48] A. Chamseddine, R. Arnowitt and P. Nath, Phys. Rev. Lett. 49 (1982) 970;

[49] L. Ibañez and G. Ross, Phys. Lett. B110 (1982) 215;

[50] R. Barbieri, S. Ferrara and C. Savoy, Phys. Lett. B119 (1982) 343.

[51] L.J. Hall, J. Lykken and S. Weinberg, Phys. Rev. D27 (1983) 2359.

[52] M. M. El Kheishen, A. A. Aboshousha and A. A. Shafik, Phys. Rev. D 45 (1992) 4345.

[53] V. Barger, M. S. Berger and P. Ohmann, Phys. Rev. D 49, 4908 (1994).

[54] G. Jungman and M. Kamionkowski, Phys. Rev. D 51, 328 (1995).

[55] J. Edsjo, Nucl. Phys. Proc. Suppl. 43, 265 (1995); Ph.D. Thesis, Uppsala University (1993).

[56] L. Bergstrom, Rept. Prog. Phys. 63, 793 (2000).

[57] H. Baer, C. Chen, M. Drees, F. Paige and X. Tata, Phys. Rev. Lett. 79, 986 (1997).

[58] M. Drees and M. Nojiri, Phys. Rev. D 47, 376 (1993).

[59] R. Arnowitt and P. Nath, Phys. Rev. D 54, 2374 (1996).

[60] H. Baer and M. Brhlik, Phys. Rev. D 53, 597 (1996); Phys. Rev. D 57, 567 (1998).

[61] V. Barger and C. Kao, Phys. Rev. D 57, 3131 (1998).

[62] J. L. Feng, K. T. Matchev and F. Wilczek, Phys. Lett. B 482, 388 (2000).

[63] J. Ellis, T. Falk, K. A. Olive and M. Srednicki, Astropart. Phys. 13, 181 (2000); J. Ellis, T. Falk, G. Ganis, K. A. Olive and M. Srednicki, CERN-TH-2001-024 (2001), hep-ph/0102098.

[64] M.S. Alam et al., the CLEO Collaboration, Phys. Rev. Lett. 74 (1995) 2885; S. Ahmed et al., [CLEO Collaboration], hep-ex/9908022.

[65] R. Barate et al., the ALEPH Collaboration, Phys. Lett. B 429, 169 (1998). 
[66] S. Bertolini, F. Borzumati and A. Masiero, Nucl. Phys. B294, 321 (1987); S. Bertolini, F. Borzumati, A. Masiero and G. Ridolfi, Nucl. Phys. B353, 591 (1991).

[67] H. Baer, M. Brhlik, D. Castano and X. Tata, Phys. Rev. D 58, 015007 (1998); and references therein.

[68] P. Nath and R. Arnowitt, Phys. Lett. B336, 395 (1994); Phys. Rev. D54, 2374 (1996).

[69] V. Barger, M.S. Berger, P. Ohmann and R.J. Phillips, Phys. Rev. D51, 2438 (1995).

[70] H. N. Brown et al. (Muon $g-2$ Collaboration), Phys. Rev. Lett. 86, 2227 (2001).

[71] J. Lopez, D. Nanopoulos and X. Wang, Phys. Rev. D49, 366 (1994);

[72] U. Chattopadhyay and P. Nath, Phys. Rev. D53, 1648 (1996).

[73] T. Moroi, Phys. Rev. D53, 6565 (1996).

[74] M. Carena, G. Giudice and C. Wagner, Phys. Lett. B390, 234 (1997);

[75] U. Chattopadhyay, D.K. Ghosh and S. Roy, Phys. Rev. D62, 115001 (2000).

[76] G. Cho, K. Hagiwara and M. Hayakawa, Phys. Lett. B 478, 231 (2000)

[77] L. Everett, G. Kane, S. Rigolin and L. Wang, hep-ph/0102145 (2001).

[78] J. Feng and K. Matchev, hep-ph/0102146 (2001).

[79] E. Baltz and P. Gondolo, hep-ph/0102147 (2001).

[80] U. Chattopadhyay and P. Nath, hep-ph/0102157 (2001).

[81] S. Komine, T. Moroi and M. Yamaguchi, hep-ph/0102204 (2001).

[82] J. Hisano and K. Tobe, hep-ph/0102315 (2001).

[83] J. Ellis, D. Nanopoulos and K. Olive, hep-ph/0102331 (2001).

[84] R. Arnowitt, B. Dutta, B. Hu and Y. Santoso, hep-ph/0102344 (2001).

[85] M. Einhorn and J. Wudka, hep-ph/0103034 (2001).

[86] K. Choi, K. Hwang, S. Kang K. Lee and W. Song, hep-ph/0103048 (2001).

[87] J. E. Kim, B. Kyae and H. M. Lee, hep-ph/0103054 (2001).

[88] S. Martin and J. D. Wells, hep-ph/0103067 (2001).

[89] H. Baer, C. Balazs, J. Ferrandis and X. Tata, Florida State University Report FSUHEP-030101 (2001), hep-ph/0103280.

[90] R. Hempfling and A.H. Hoang, Phys. Lett. B 331, 99 (1994); H. Haber, R. Hempfling and A. Hoang, Z. Phys. C75, 539 (1997).

[91] M. Carena, M. Quiros, C.E.M. Wagner, Nucl. Phys. B461, 407 (1996).

[92] S. Heinemeyer, W. Hollik, G. Weiglein, Phys. Rev. D 58, 091701 (1998).

[93] R.-J. Zhang, Phys. Lett. B 447, 89 (1999).

[94] M. Carena, H. E. Haber, S. Heinemeyer, W. Hollik, C. E. Wagner and G. Weiglein, Nucl. Phys. B580 (2000) 29.

[95] J. R. Espinosa and R. Zhang, JHEP 0003 (2000) 026; Nucl. Phys. B 586 (2000) 3.

[96] T. Falk, Phys. Lett. B 456, 171 (1999).

[97] J. L. Feng, K. T. Matchev and F. Wilczek, Phys. Lett. B 482, 388 (2000).

[98] The LEP2 SUSY working group, http://www.cern.ch/lepsusy/, Charginos, large m0 (LEPSUSYWG/01-03.1).

[99] J. L. Feng, K. T. Matchev and T. Moroi, Phys. Rev. Lett. 84, 2322 (2000); J. L. Feng, K. T. Matchev and T. Moroi, Phys. Rev. D 61, 075005 (2000)

[100] V. Berezinsky, A. Bottino, J. Ellis, N. Fornengo, G. Mignola and S. Scopel, Astropart. Phys. 5, 1 (1996)

[101] P. Nath and R. Arnowitt, Phys. Rev. D 56, 2820 (1997). 


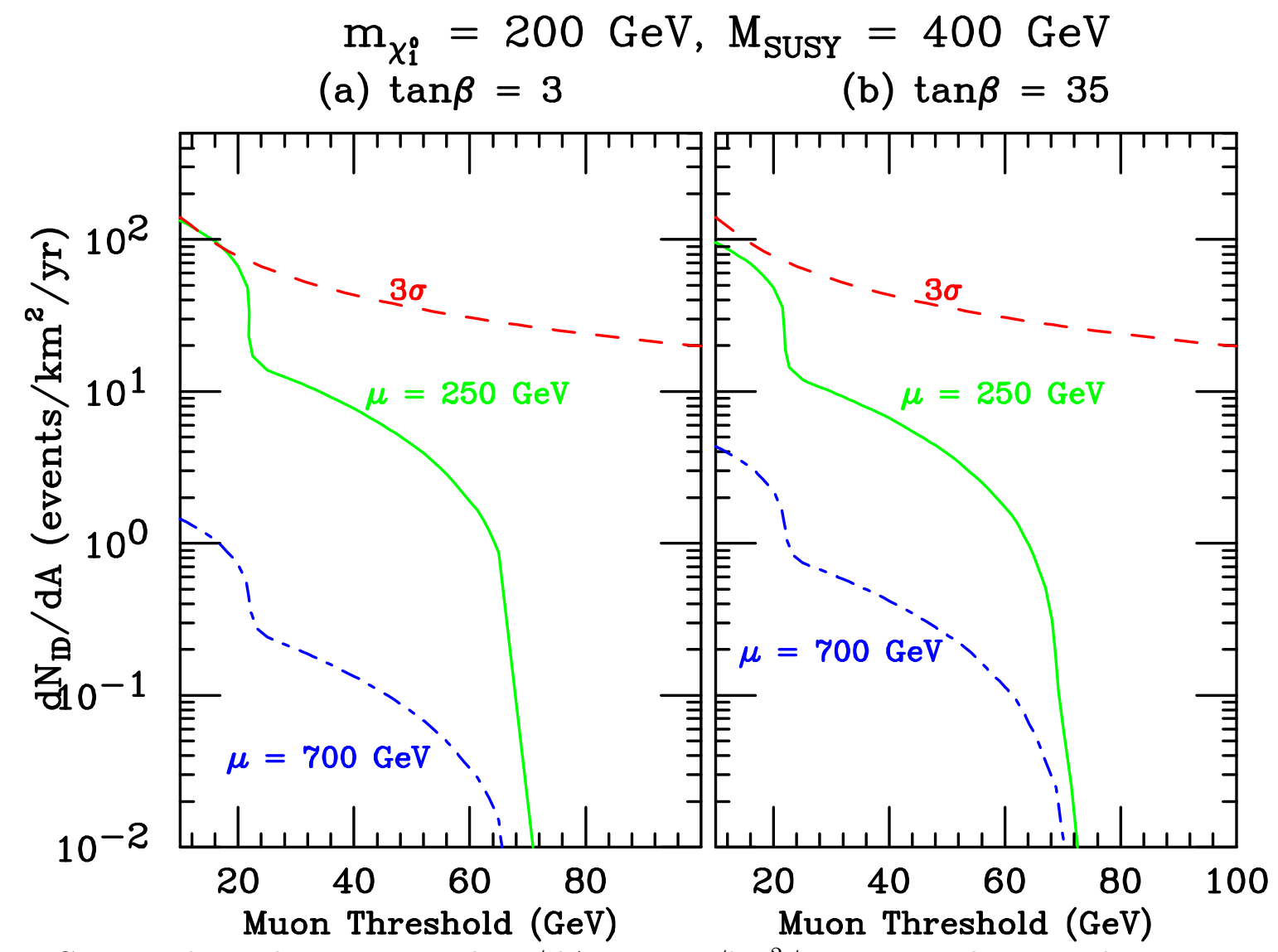

FIG. 1. Indirect detection rate $d N_{\mathrm{ID}} / d A$ in events $/ \mathrm{km}^{2} /$ year versus the muon detection energy threshold for $m_{\chi_{1}^{0}}=200 \mathrm{GeV}$ and $M_{\mathrm{SUSY}}=400 \mathrm{GeV}$ with $m_{A}=175 \mathrm{GeV}$ and (a) $\tan \beta=3$, $\mu=700 \mathrm{GeV}, M_{2}=400 \mathrm{GeV}$, and $\mu=250 \mathrm{GeV}, M_{2}=485 \mathrm{GeV}$, as well as (b) $\tan \beta=35$, $\mu=700 \mathrm{GeV}, M_{2}=400 \mathrm{GeV}$, and $\mu=250 \mathrm{GeV}, M_{2}=445 \mathrm{GeV}$. Also shown is the $3 \sigma$ signal rate $\left(=3 \sqrt{N_{B}}\right)$, where $N_{B}$ is the number of background events from the atmospheric neutrinos. 


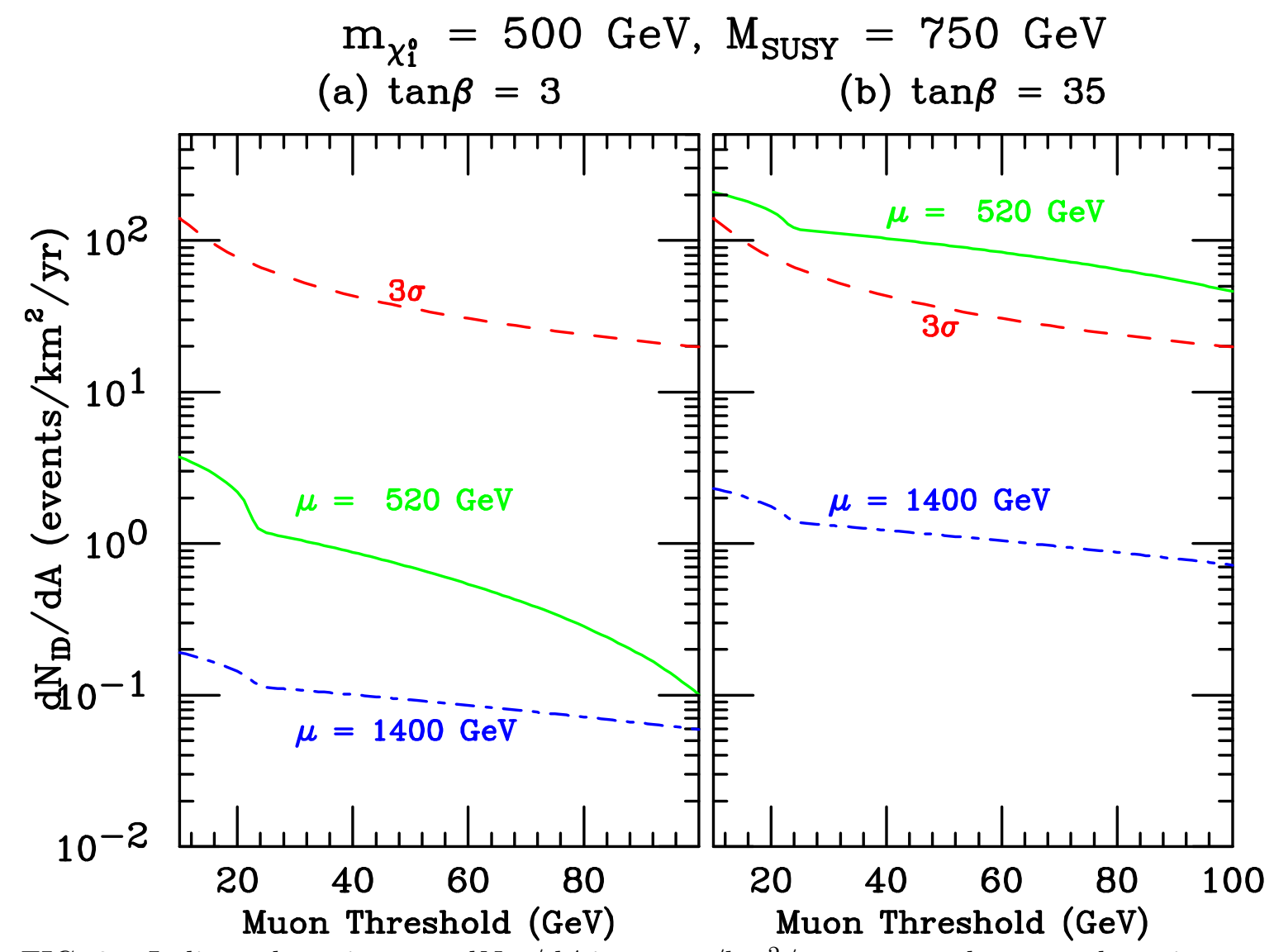

FIG. 2. Indirect detection rate $d N_{\mathrm{ID}} / d A$ in events $/ \mathrm{km}^{2} /$ year versus the muon detection energy threshold for $m_{\chi_{1}^{0}}=500 \mathrm{GeV}$ and $M_{\mathrm{SUSY}}=750 \mathrm{GeV}$ with $m_{A}=500 \mathrm{GeV}$ and (a) $\tan \beta=3$, $\mu=1400 \mathrm{GeV}, M_{2}=1000 \mathrm{GeV}$, and $\mu=520 \mathrm{GeV}, M_{2}=1280 \mathrm{GeV}$, as well as (b) $\tan \beta=35$, $\mu=1400 \mathrm{GeV}, M_{2}=1000 \mathrm{GeV}$, and $\mu=520 \mathrm{GeV}, M_{2}=1160 \mathrm{GeV}$. Also shown is the $3 \sigma$ signal rate $\left(=3 \sqrt{N_{B}}\right)$, where $N_{B}$ is the number of background events from the atmospheric neutrinos. 


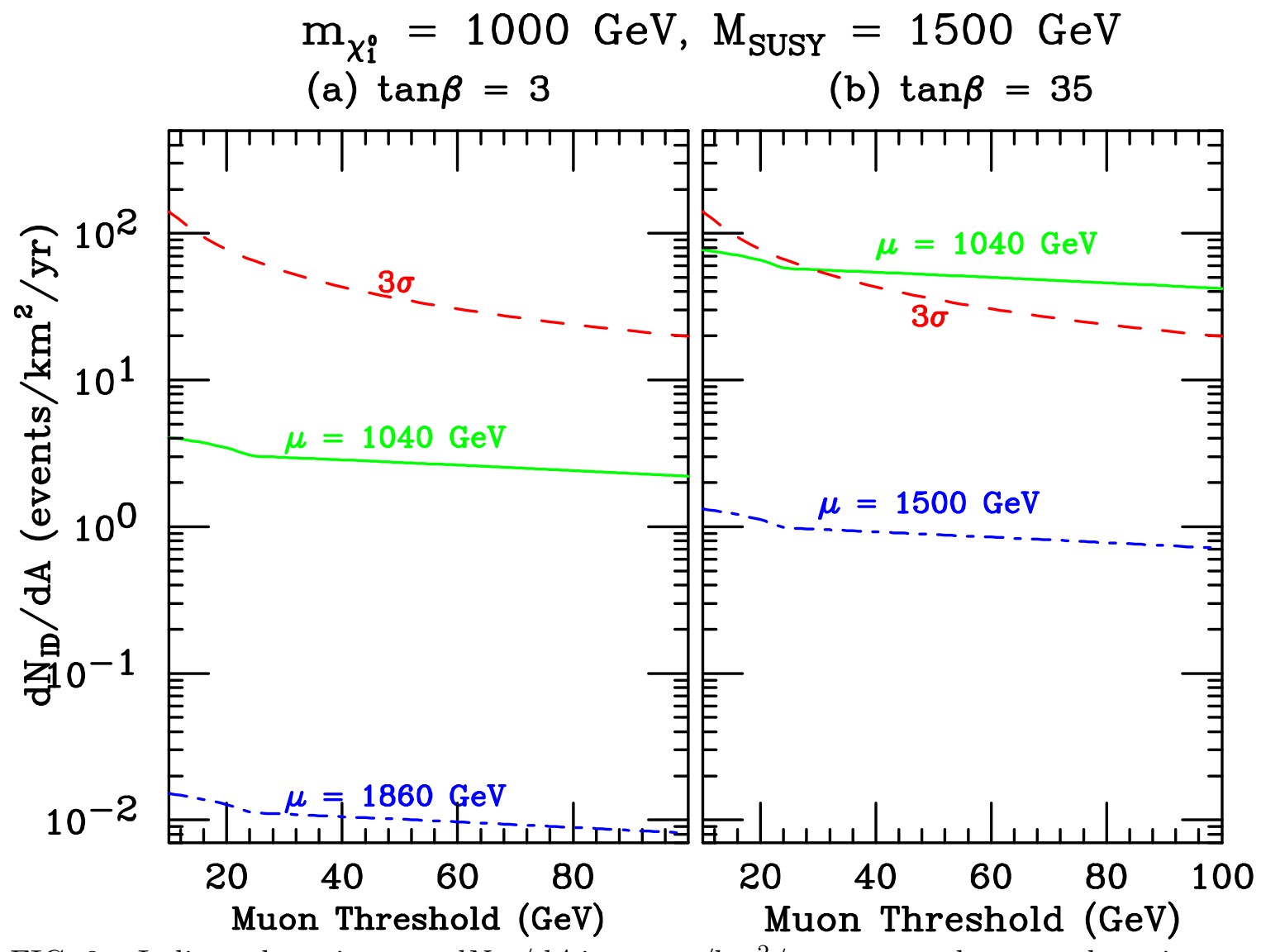

FIG. 3. Indirect detection rate $d N_{\mathrm{ID}} / d A$ in events $/ \mathrm{km}^{2} /$ year versus the muon detection energy threshold for $m_{\chi_{1}^{0}}=1000 \mathrm{GeV}$ and $M_{\mathrm{SUSY}}=1500 \mathrm{GeV}$ with $m_{A}=1000 \mathrm{GeV}$ and (a) $\tan \beta=3$, $\mu=1040 \mathrm{GeV}, M_{2}=2060 \mathrm{GeV}$, and $\mu=1860 \mathrm{GeV}, M_{2}=2000 \mathrm{GeV}$, as well as (b) $\tan \beta=35$, $\mu=1040 \mathrm{GeV}, M_{2}=2060 \mathrm{GeV}$, and $\mu=1500 \mathrm{GeV}, M_{2}=2000 \mathrm{GeV}$. Also shown is the $3 \sigma$ signal rate $\left(=3 \sqrt{N_{B}}\right)$, where $N_{B}$ is the number of background events from the atmospheric neutrinos. 


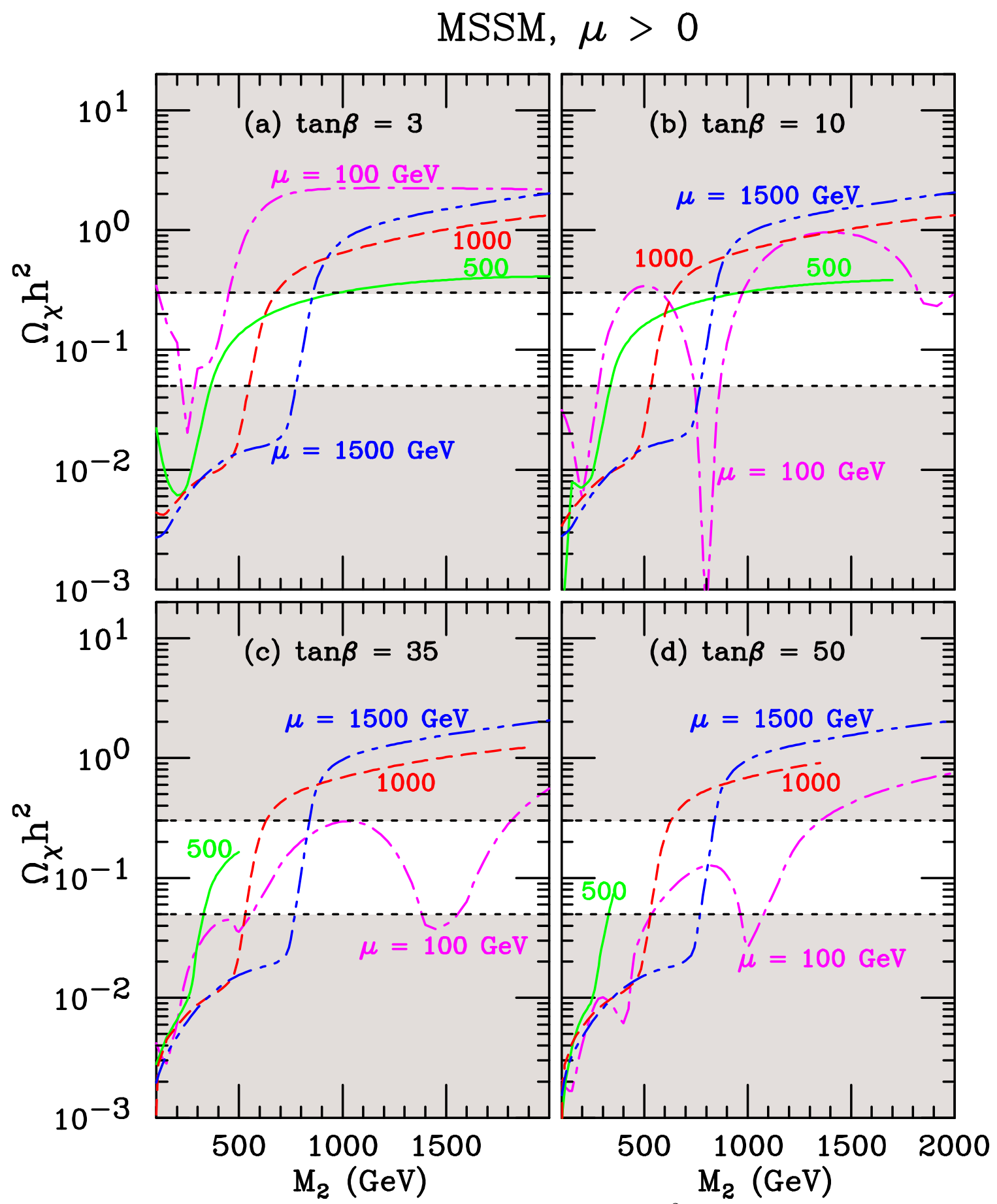

FIG. 4. Relic density of the neutralino dark matter $\left(\Omega_{\chi_{1}^{0}} h^{2}\right)$ versus $M_{2}$ in the MSSM with several values of $\mu$ and $M_{\mathrm{SUSY}}=\operatorname{MAX}\left(300 \mathrm{GeV}, 1.5 m_{\chi_{1}^{0}}\right)$ for (a) $\tan \beta=3$, (b) $\tan \beta=10$, (c) $\tan \beta=35$ and $(\mathrm{d}) \tan \beta=50$. 


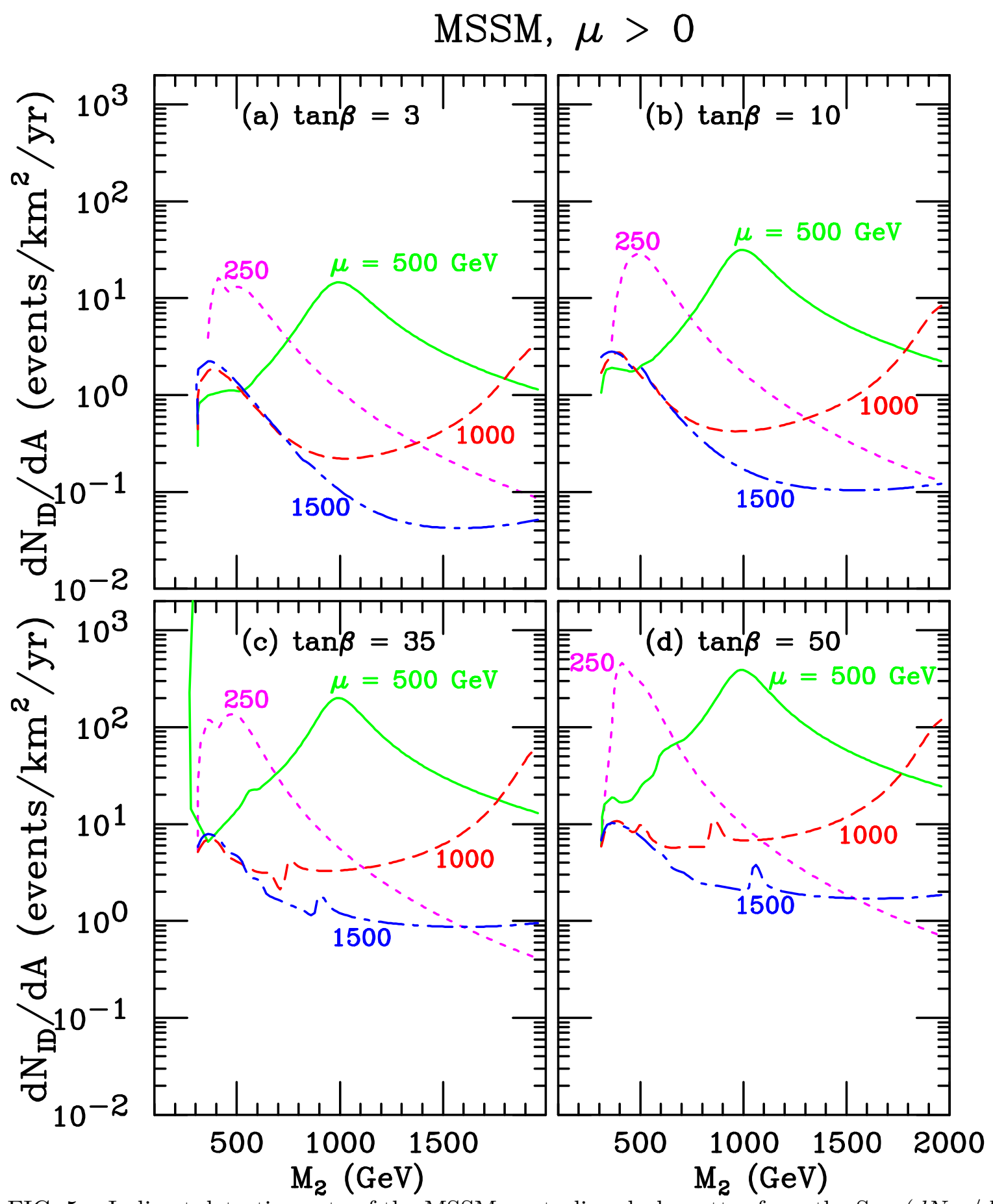

FIG. 5. Indirect detection rate of the MSSM neutralino dark matter from the Sun $\left(d N_{\mathrm{ID}} / d A\right)$ in Events $/ \mathrm{km}^{2} /$ year versus $M_{2}$ with $M_{\mathrm{SUSY}}=\operatorname{MAX}\left(300 \mathrm{GeV}, 1.5 m_{\chi_{1}^{0}}\right)$ and several values of $\mu$ for (a) $\tan \beta=3$, (b) $\tan \beta=10$, (c) $\tan \beta=35$ and (d) $\tan \beta=50$. 


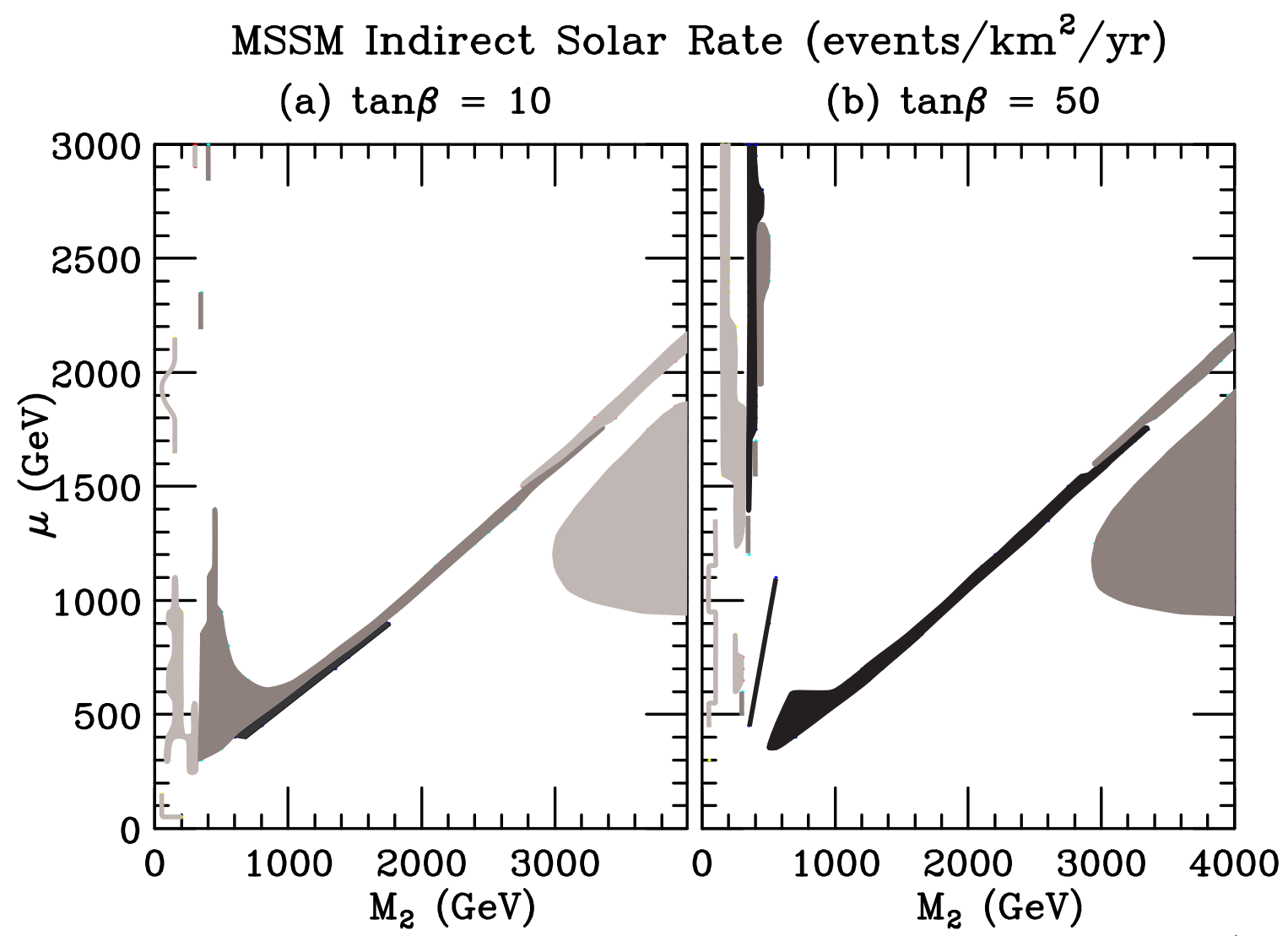

FIG. 6. Regions of indirect detection rate of the MSSM neutralino dark matter in the $\left(M_{2}, \mu\right)$ plane with $M_{\text {SUSY }}=\operatorname{MAX}\left(300 \mathrm{GeV}, 1.5 m_{\chi_{1}^{0}}\right)$ for (a) $\tan \beta=10$ and (b) $\tan \beta=50$. The region with dark shading has $d N_{\mathrm{ID}} / d A>100$; the region with light shading has $d N_{\mathrm{ID}} / d A<10$; and the region with intermediate shading has $100>d N_{\mathrm{ID}} / d A>10$ (event $/ \mathrm{km}^{2} /$ year). The blank regions do not have a cosmologically interesting relic density $\left(0.05 \leq \Omega_{\chi_{1}^{0}} \leq 0.3\right)$ for the neutralino dark matter. 


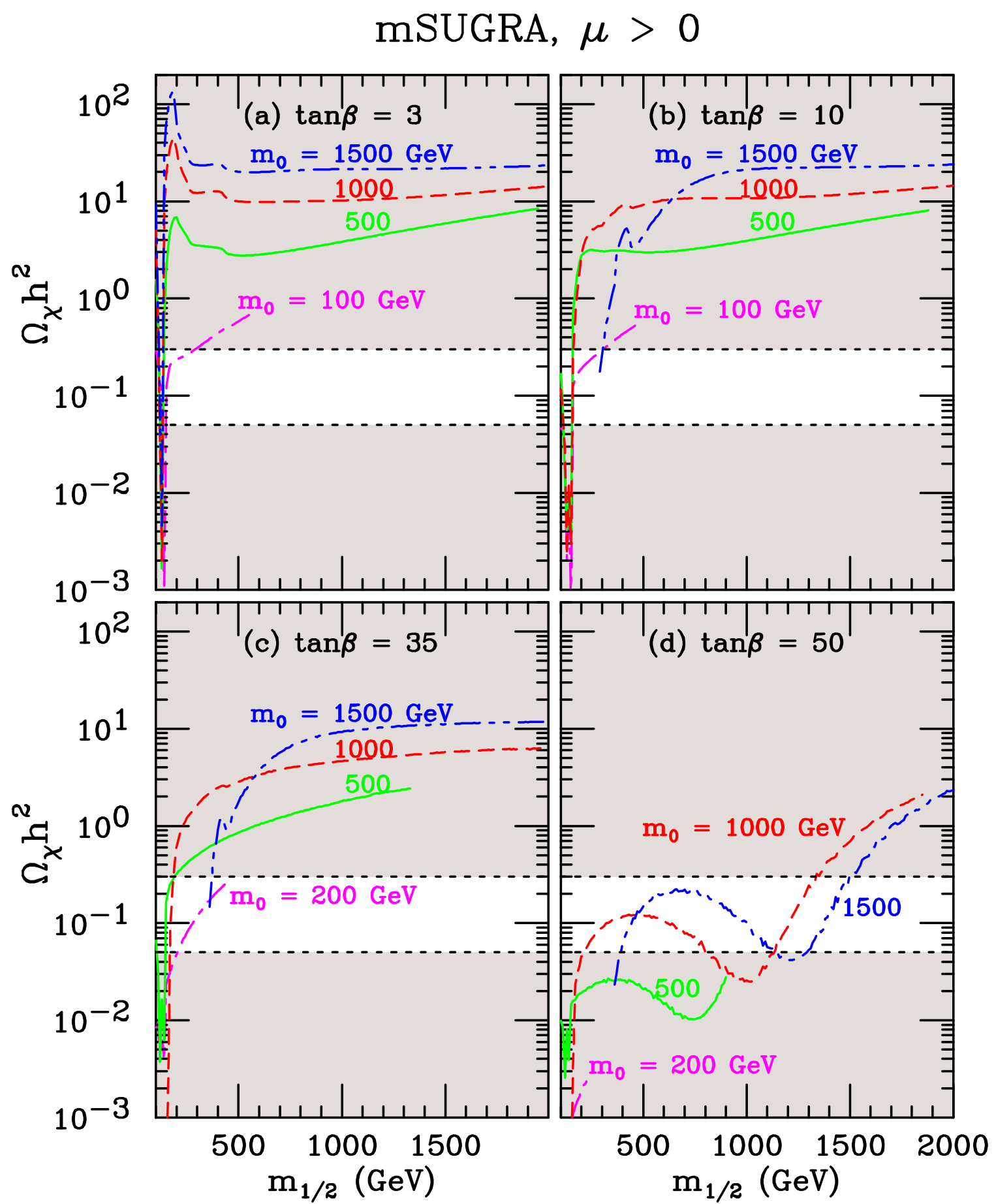

FIG. 7. Relic density of the neutralino dark matter $\left(\Omega_{\chi_{1}^{0}} h^{2}\right)$ versus $m_{1 / 2}$ in the mSUGRA model with $\mu>0, A_{0}=0$ and several values of $m_{0}$ for (a) $\tan \beta=3$, (b) $\tan \beta=10$, (c) $\tan \beta=35$ and (d) $\tan \beta=50$. 


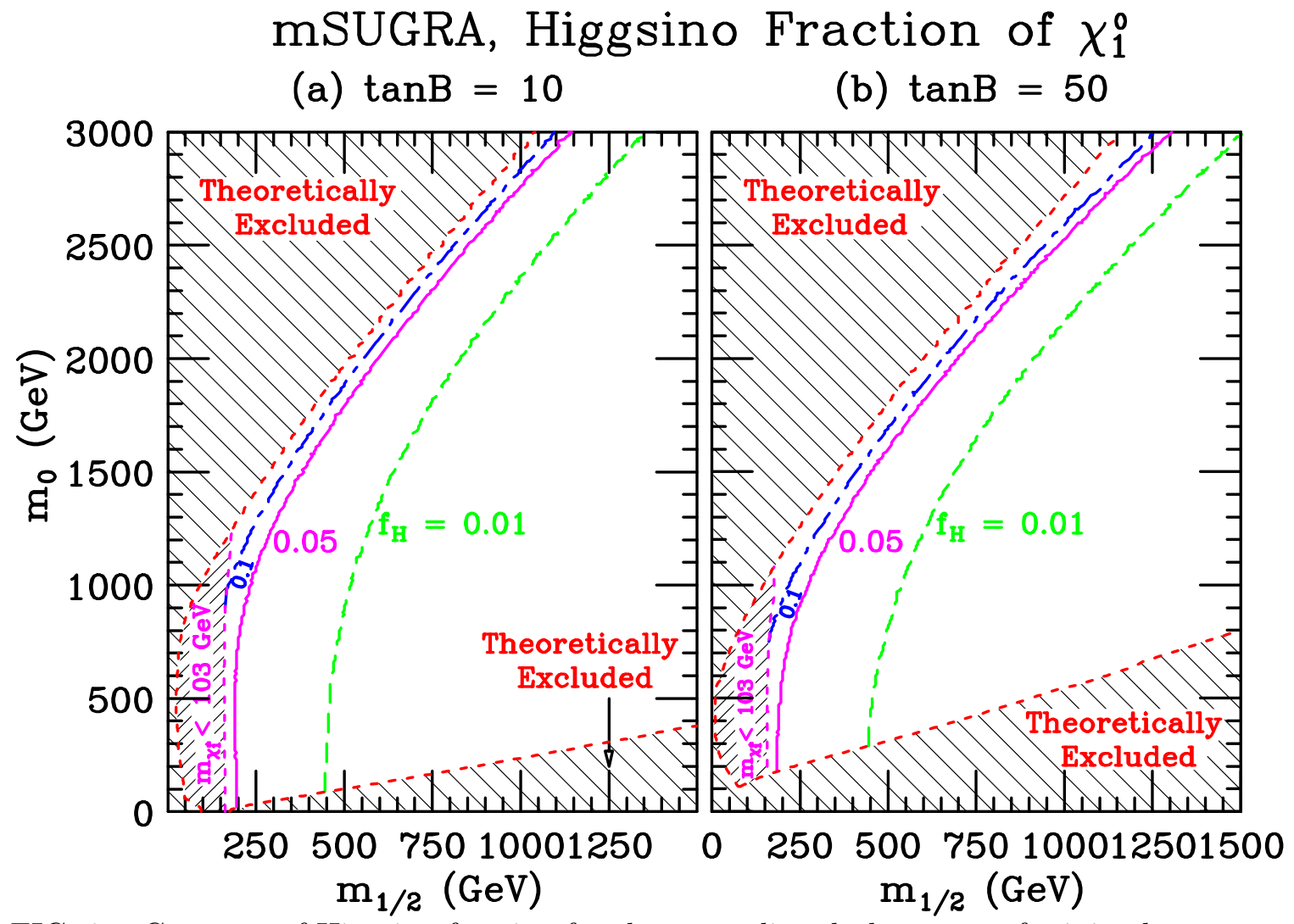

FIG. 8. Contours of Higgsino fraction for the neutralino dark matter of minimal supergravity in the $\left(m_{1 / 2}, m_{0}\right)$ plane for (a) $\tan \beta=10$ and (b) $\tan \beta=50$. Also shown are the parts of the parameter space (i) excluded by theoretical requirements, or (ii) excluded by the chargino search at LEP 2. 


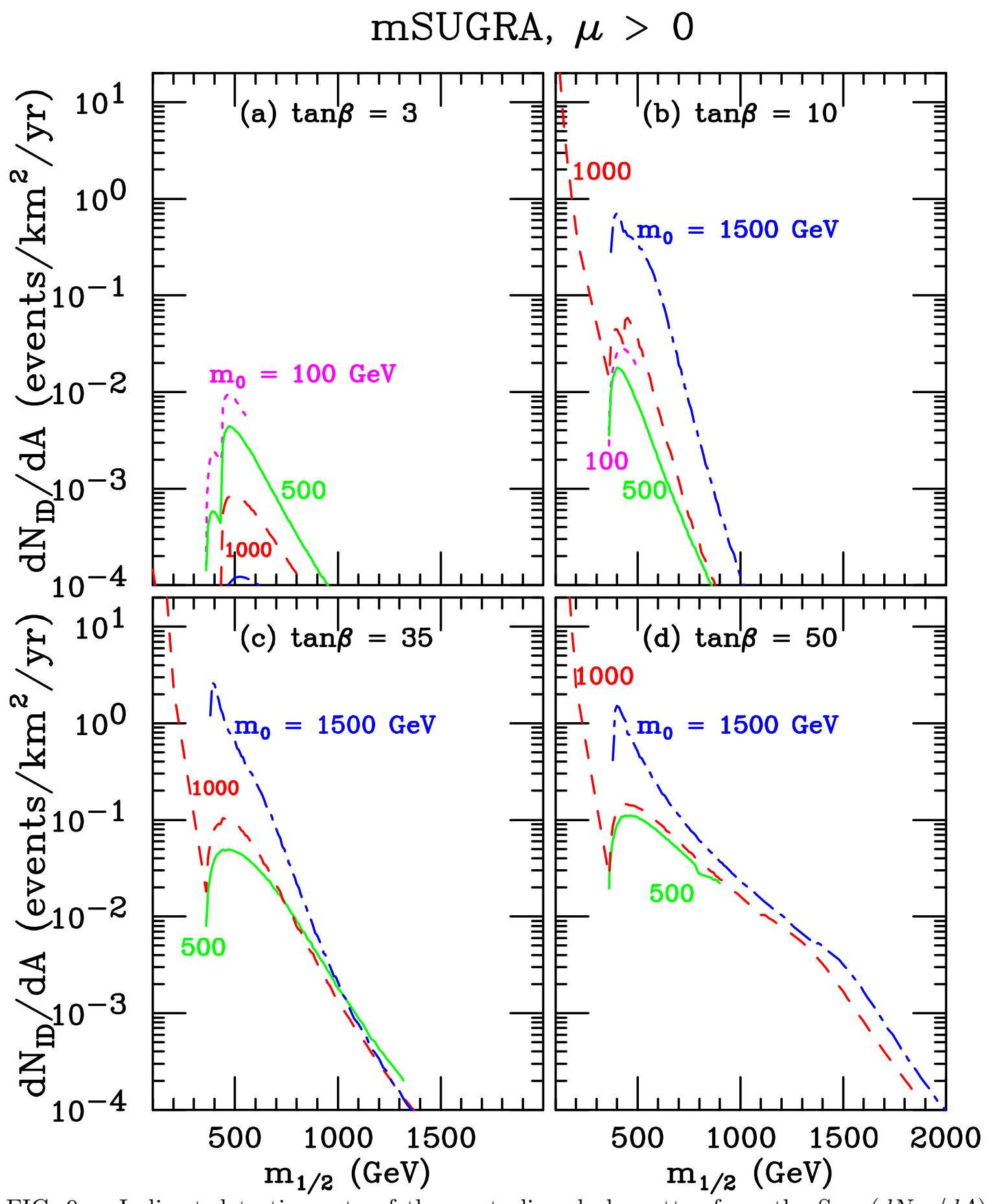

FIG. 9. Indirect detection rate of the neutralino dark matter from the Sun $\left(d N_{\mathrm{ID}} / d A\right)$ in Events $/ \mathrm{km}^{2} /$ year versus $m_{1 / 2}$ with $\mu>0, A_{0}=0$ and several values of $m_{0}$ for (a) $\tan \beta=3$, (b) $\tan \beta=10$, (c) $\tan \beta=35$ and (d) $\tan \beta=50$. 


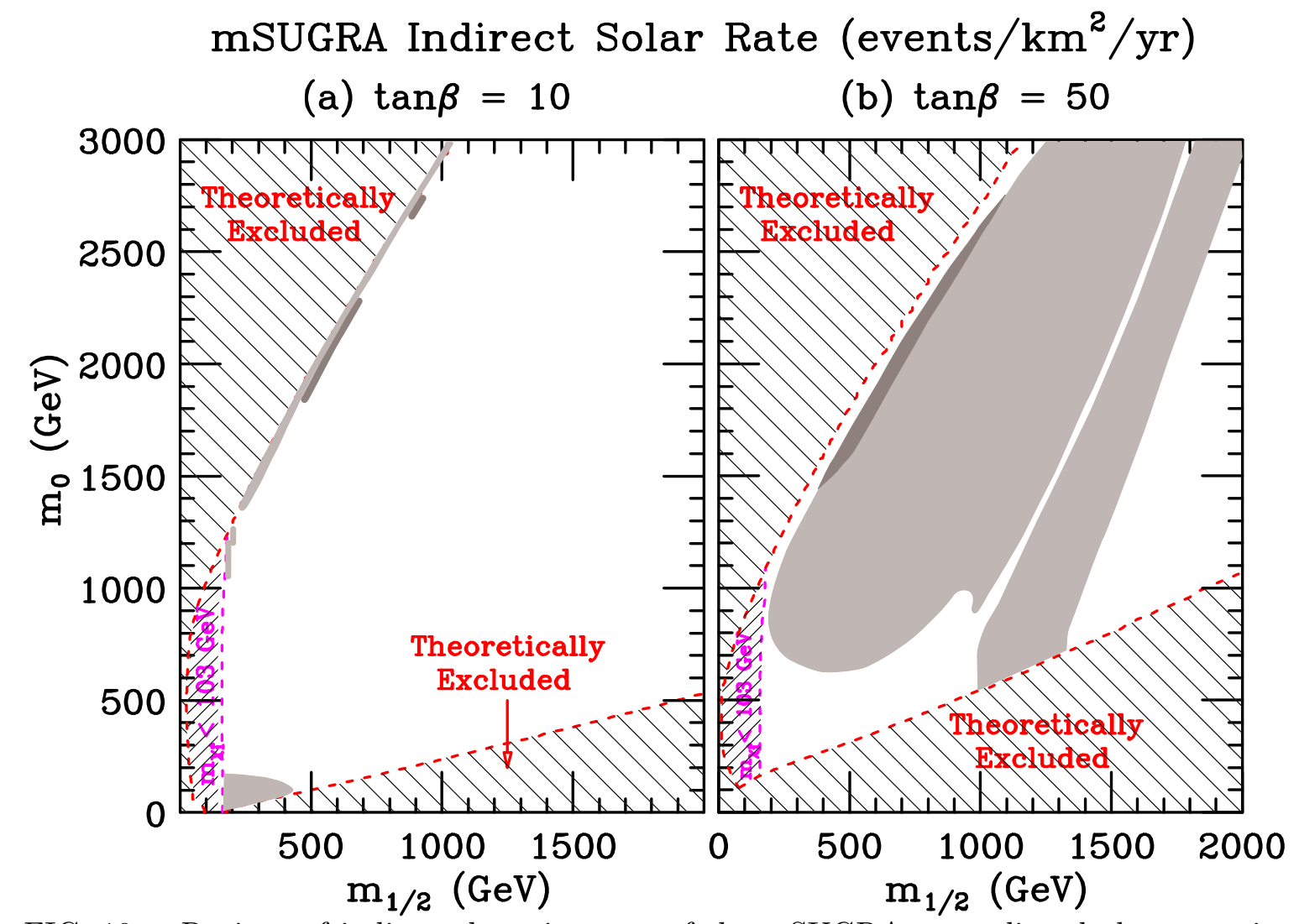

FIG. 10. Regions of indirect detection rate of the mSUGRA neutralino dark matter in the $\left(m_{1 / 2}, m_{0}\right)$ plane with $\mu>0$ and $A_{0}=0$ for (a) $\tan \beta=10$ and (b) $\tan \beta=50$. The region with light shading has $d N_{\mathrm{ID}} / d A<1$; and the region with intermediate shading has $10>d N_{\mathrm{ID}} / d A>1$ (event $/ \mathrm{km}^{2} /$ year). There is no region with dark shading that has $d N_{\mathrm{ID}} / d A>10$. The blank regions do not have a cosmologically interesting relic density $\left(0.05 \leq \Omega_{\chi_{1}^{0}} \leq 0.3\right)$ for the neutralino dark matter. Also shown are the parts of the parameter space (i) excluded by theoretical requirements, or (ii) excluded by the chargino search at LEP 2 . 


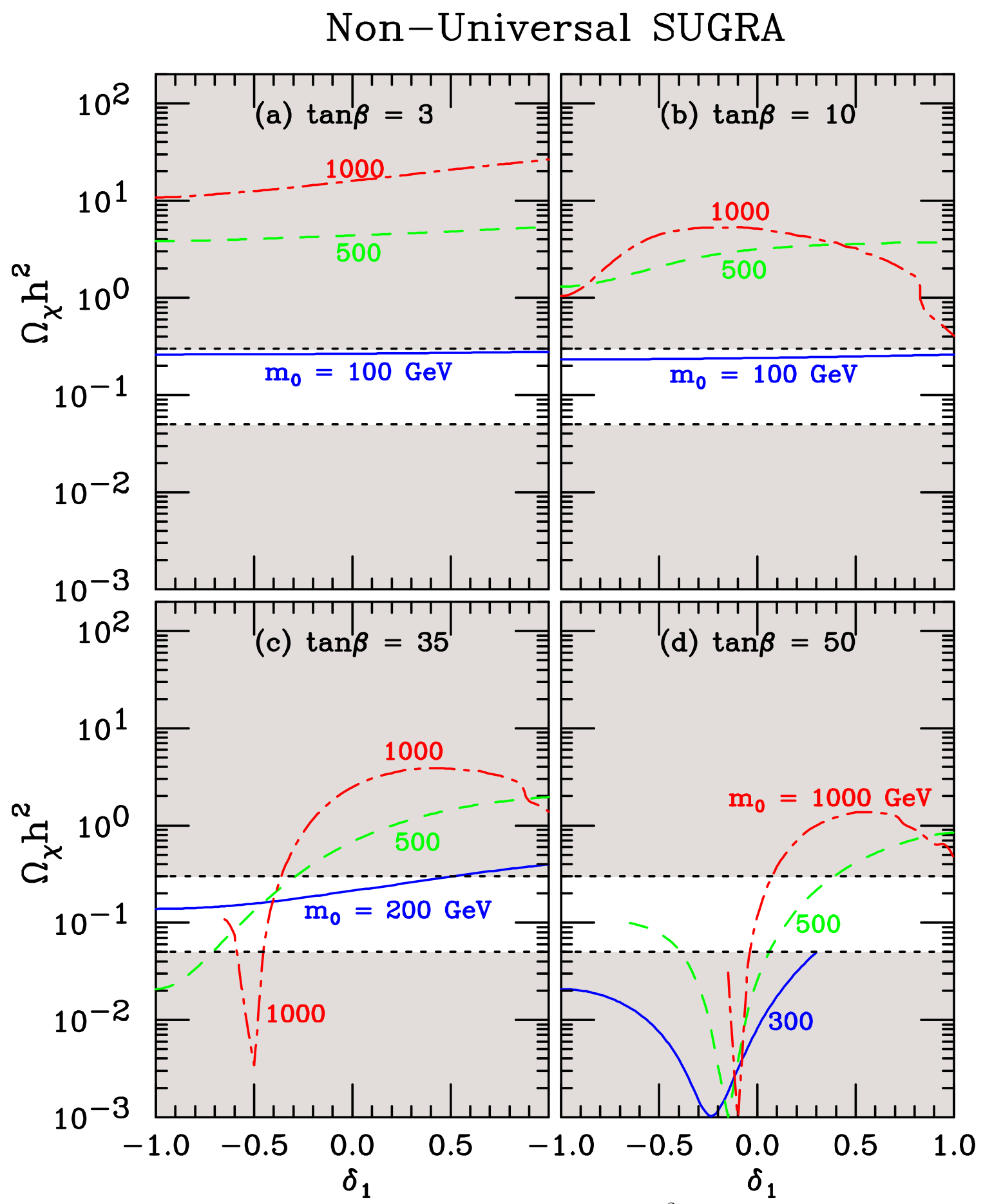

FIG. 11. Relic density of the neutralino dark matter $\left(\Omega_{\chi_{1}^{0}} h^{2}\right)$ versus $\delta_{1}$ in a SUGRA model with non-universal Higgs masses at $M_{\mathrm{GUT}}$, with $\delta_{2}=0, \mu>0, A_{0}=0$, and several values of $m_{0}$, for $m_{1 / 2}=250 \mathrm{GeV}$ with (a) $\tan \beta=3$ and (b) $\tan \beta=10$, as well as for $m_{1 / 2}=400 \mathrm{GeV}$ with (c) $\tan \beta=35$ and (d) $\tan \beta=50$. 


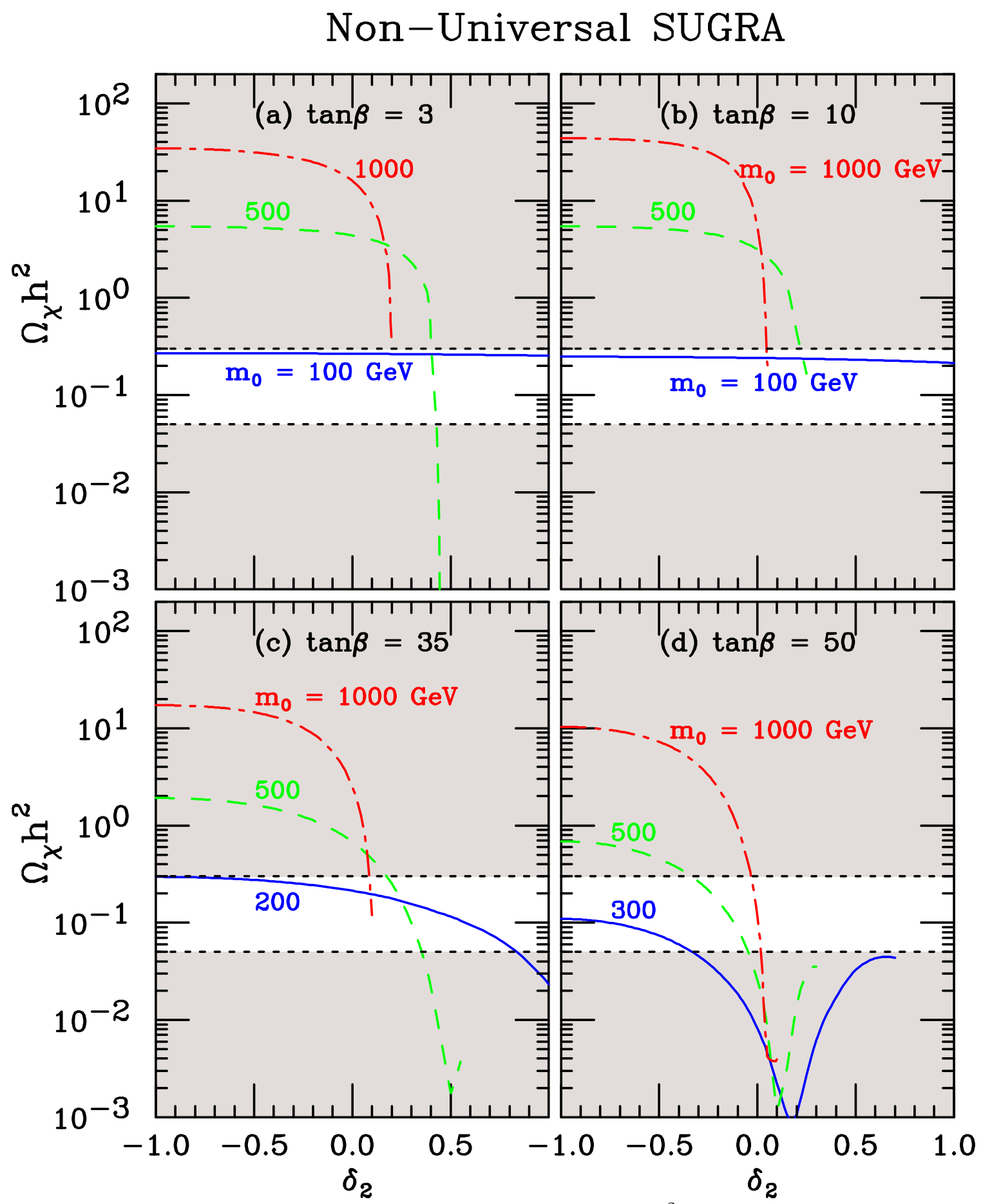

FIG. 12. Relic density of the neutralino dark matter $\left(\Omega_{\chi_{1}^{0}} h^{2}\right)$ versus $\delta_{2}$ in a SUGRA model with non-universal Higgs masses at $M_{\mathrm{GUT}}$, with $\delta_{1}=0, \mu>0, A_{0}=0$, and several values of $m_{0}$, for $m_{1 / 2}=250 \mathrm{GeV}$ with (a) $\tan \beta=3$ and (b) $\tan \beta=10$, as well as for $m_{1 / 2}=400 \mathrm{GeV}$ with (c) $\tan \beta=35$ and (d) $\tan \beta=50$. 


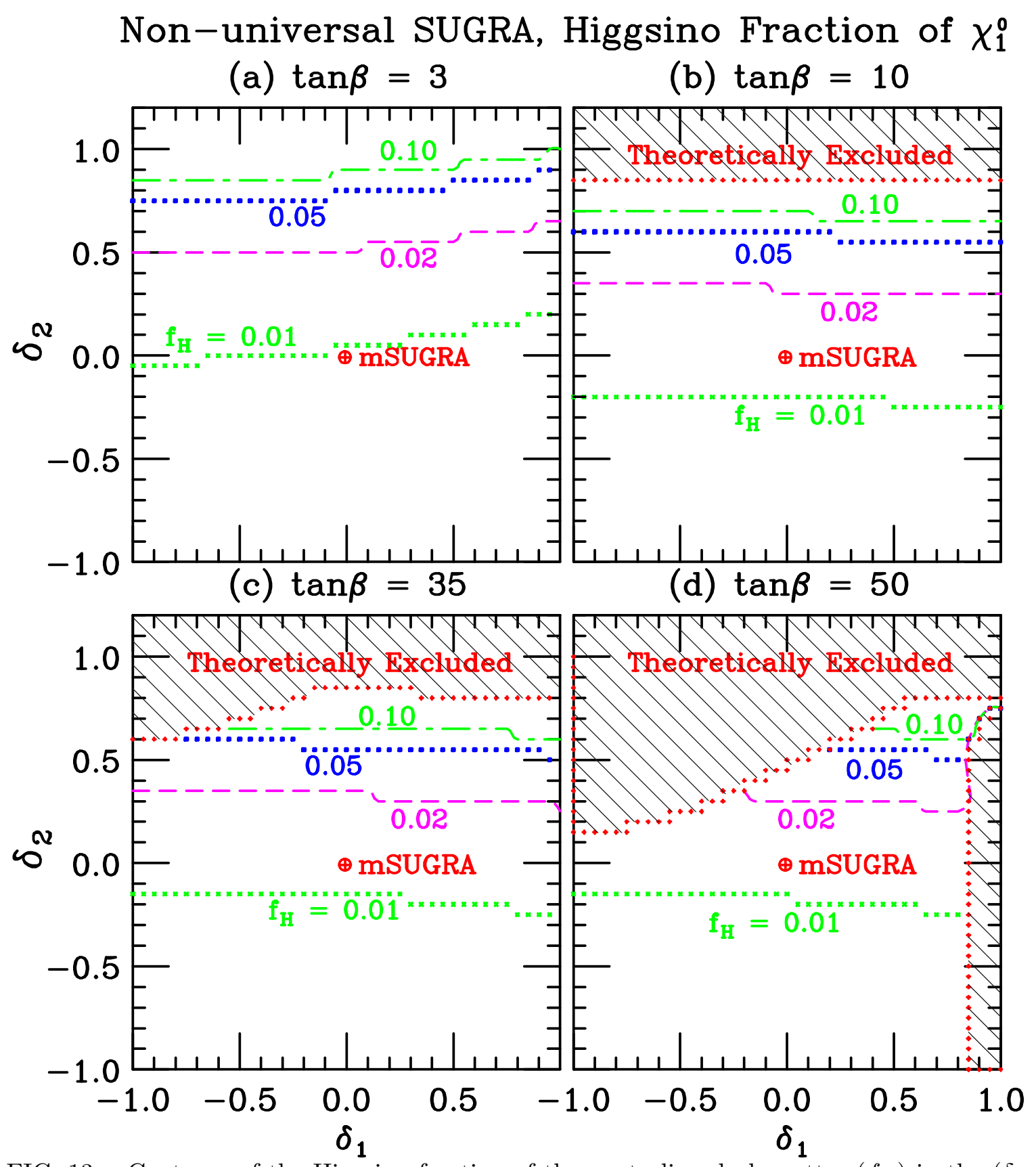

FIG. 13. Contours of the Higgsino fraction of the neutralino dark matter $\left(f_{H}\right)$ in the $\left(\delta_{1}, \delta_{2}\right)$ plan within the framework of a sugravity unified model with non-universal Higgs masses at $M_{\mathrm{GUT}}$, $\mu>0, m_{1 / 2}=m_{0}=400 \mathrm{GeV}$, for (a) $\tan \beta=3$, (b) $\tan \beta=10$, (c) $\tan \beta=35$ and (d) $\tan \beta=50$. 


\section{Non-Universal SUGRA}

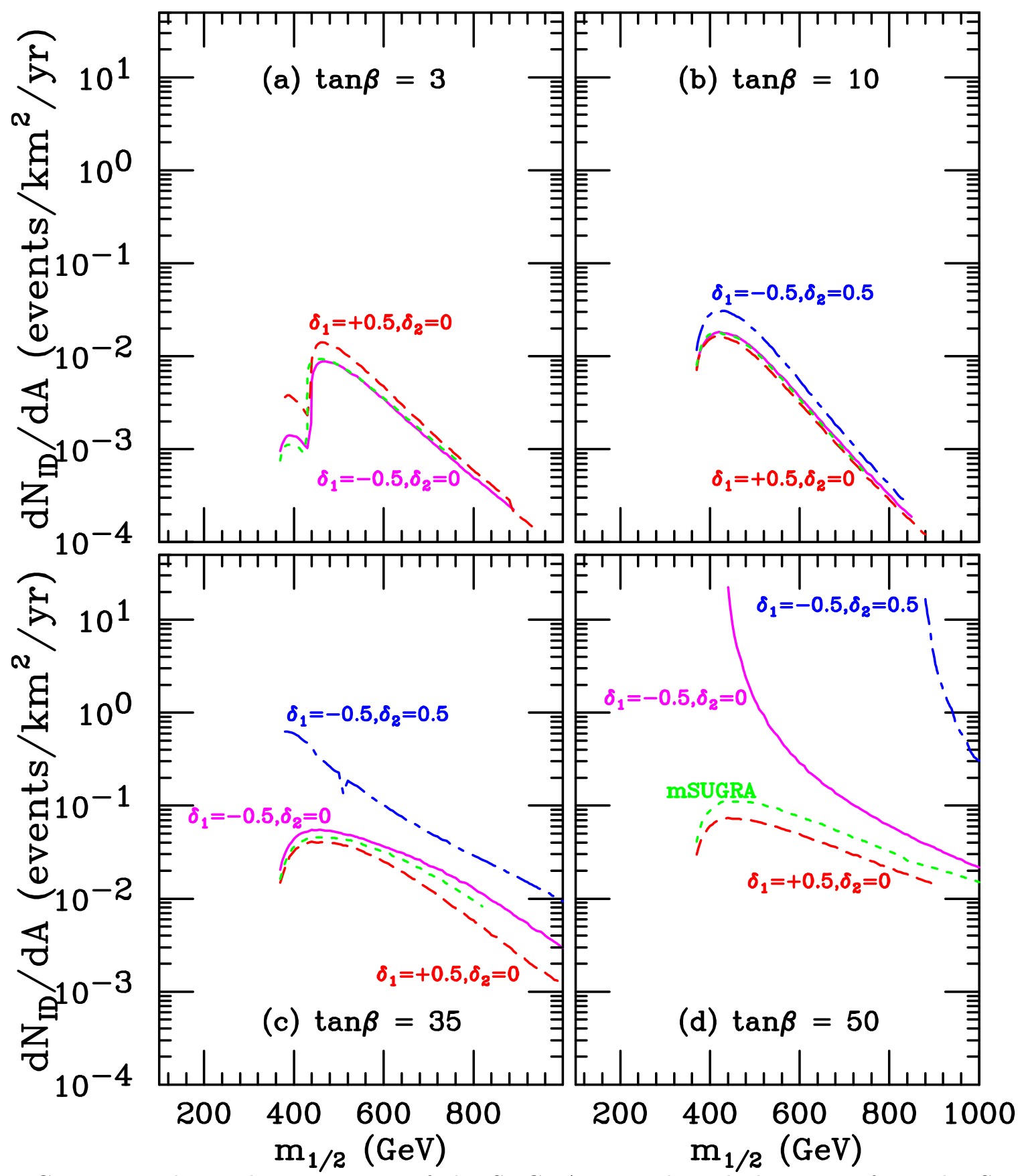

FIG. 14. Indirect detection rate of the SUGRA neutralino dark matter from the Sun in Events $/ \mathrm{km}^{2} /$ year versus $m_{1 / 2}$ with $\mu>0, A_{0}=0$, and three choices of non-universal boundary conditions for the Higgs masses at $M_{\mathrm{GUT}}$ (i) $\delta_{1}=-0.5$ and $\delta_{2}=0$, (ii) $\delta_{1}=+0.5$ and $\delta_{2}=0$, (iii) $\delta_{1}=-0.5$ and $\delta_{2}=+0.5$, for $m_{0}=200 \mathrm{GeV}$ with (a) $\tan \beta=3$, (b) $\tan \beta=10$ and (c) $\tan \beta=35$, as well as for $m_{0}=1000 \mathrm{GeV}$ with (d) $\tan \beta=50$. Also shown is the indirect detection rate in the mSUGRA with $\delta_{1}=\delta_{2}=0$. 


\section{Non-Universal SUGRA}

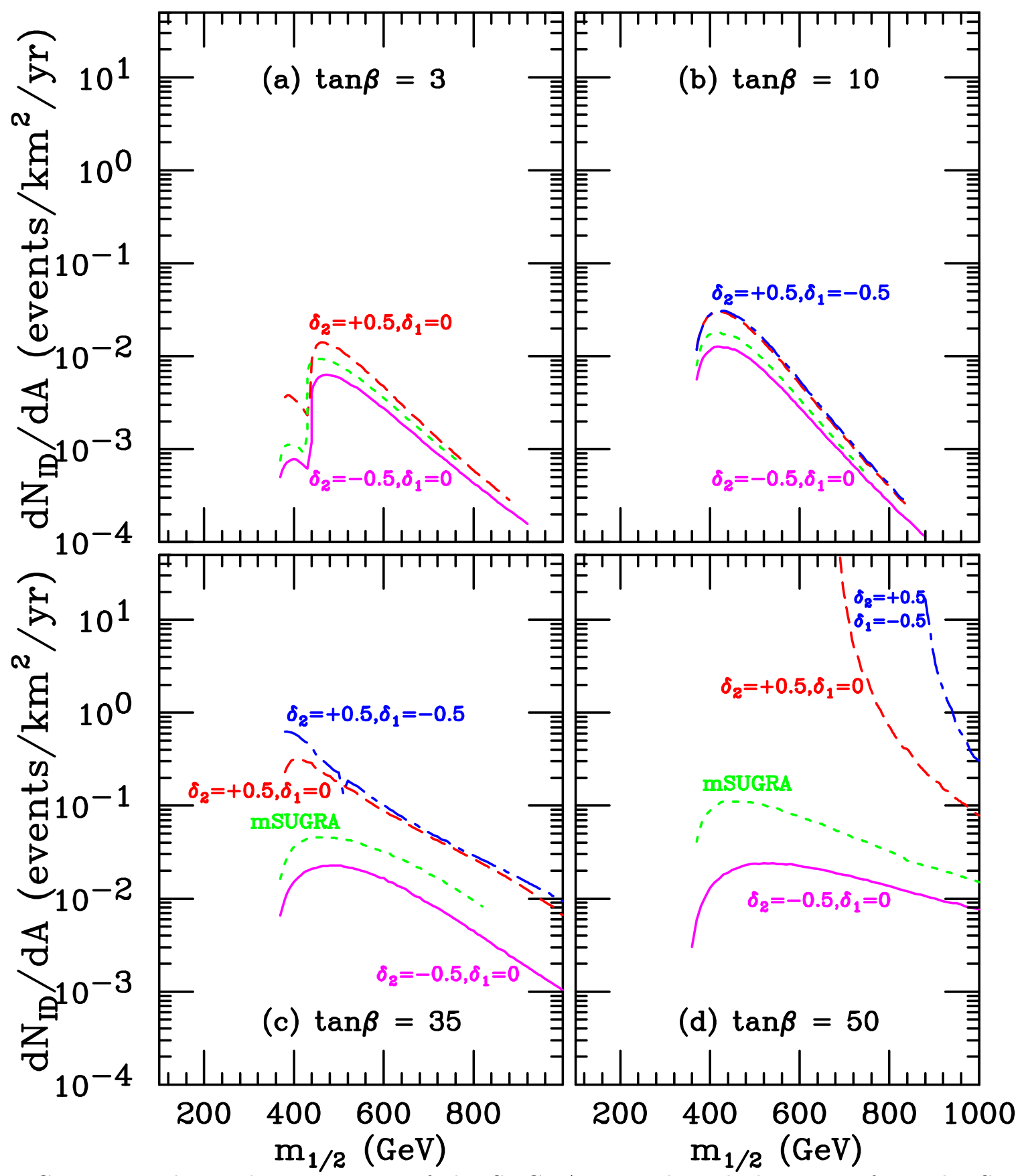

FIG. 15. Indirect detection rate of the SUGRA neutralino dark matter from the Sun in Events $/ \mathrm{km}^{2} /$ year versus $m_{1 / 2}$ with $\mu>0, A_{0}=0$, and three choices of non-universal boundary conditions for the Higgs masses at $M_{\mathrm{GUT}}$ (i) $\delta_{1}=0$ and $\delta_{2}=-0.5$, (ii) $\delta_{1}=0$ and $\delta_{2}=+0.5$, (iii) $\delta_{1}=-0.5$ and $\delta_{2}=+0.5$, for $m_{0}=200 \mathrm{GeV}$ with (a) $\tan \beta=3$, (b) $\tan \beta=10$ and (c) $\tan \beta=35$, as well as for $m_{0}=1000 \mathrm{GeV}$ with (d) $\tan \beta=50$. Also shown is the indirect detection rate in the mSUGRA with $\delta_{1}=\delta_{2}=0$. 


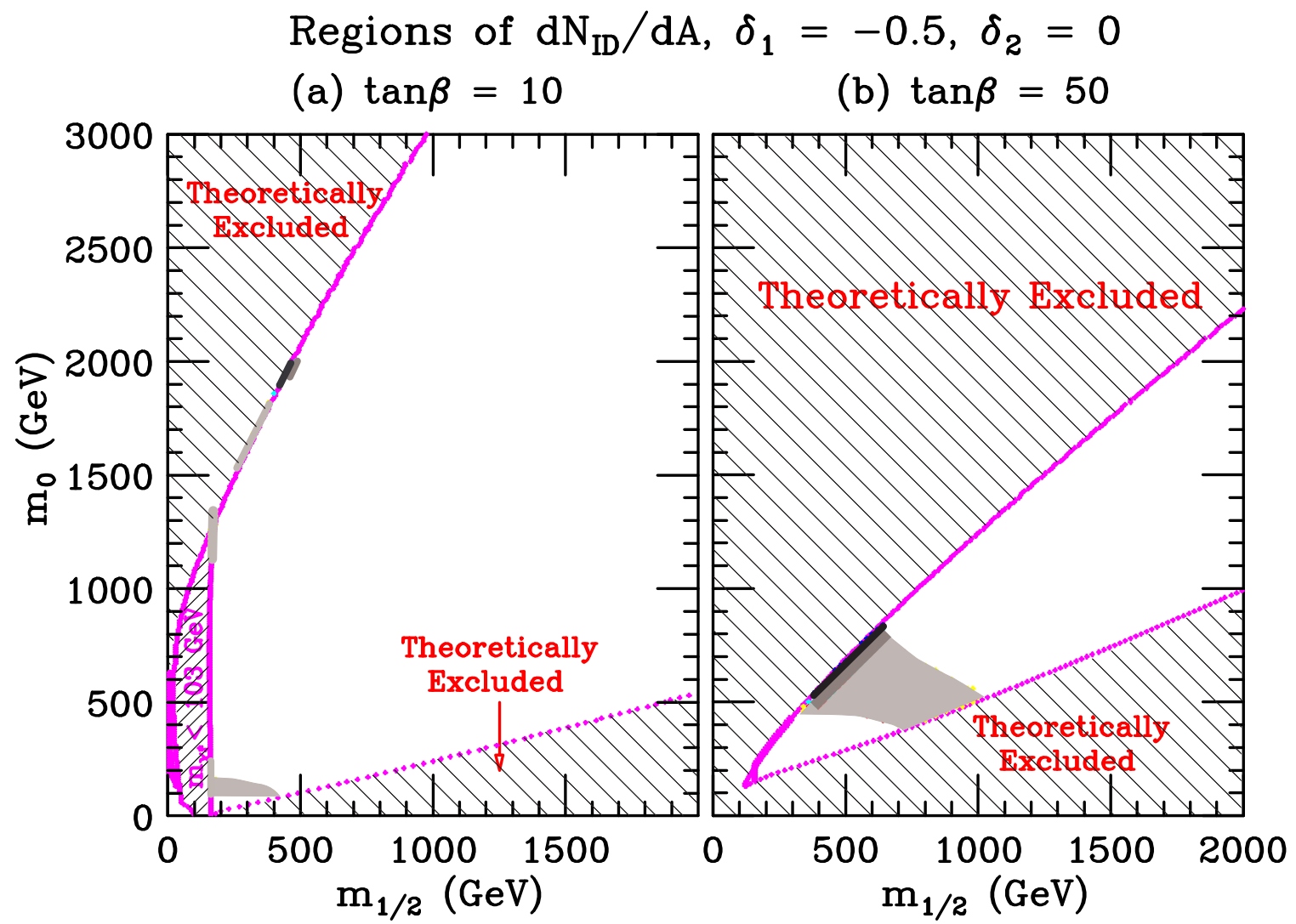

FIG. 16. Regions of indirect detection rate of the neutralino dark matter in the $\left(m_{1 / 2}, m_{0}\right)$ plane of a non-universal SUGRA model with $\mu>0, A_{0}=0$ and non-universal boundary conditions $\delta_{1}=-0.5$ and $\delta_{2}=0$, for (a) $\tan \beta=10$ and (b) $\tan \beta=50$. The region with dark shading has $d N_{\mathrm{ID}} / d A>10$; the region with light shading has $d N_{\mathrm{ID}} / d A<1$; and the region with intermediate shading has $10>d N_{\mathrm{ID}} / d A>1$ (event $/ \mathrm{km}^{2} /$ year). The blank regions do not have a cosmologically interesting relic density $\left(0.05 \leq \Omega_{\chi_{1}^{0}} \leq 0.3\right)$ for the neutralino dark matter. Also shown are the parts of the parameter space (i) excluded by theoretical requirements, or (ii) excluded by the chargino search at LEP 2. 


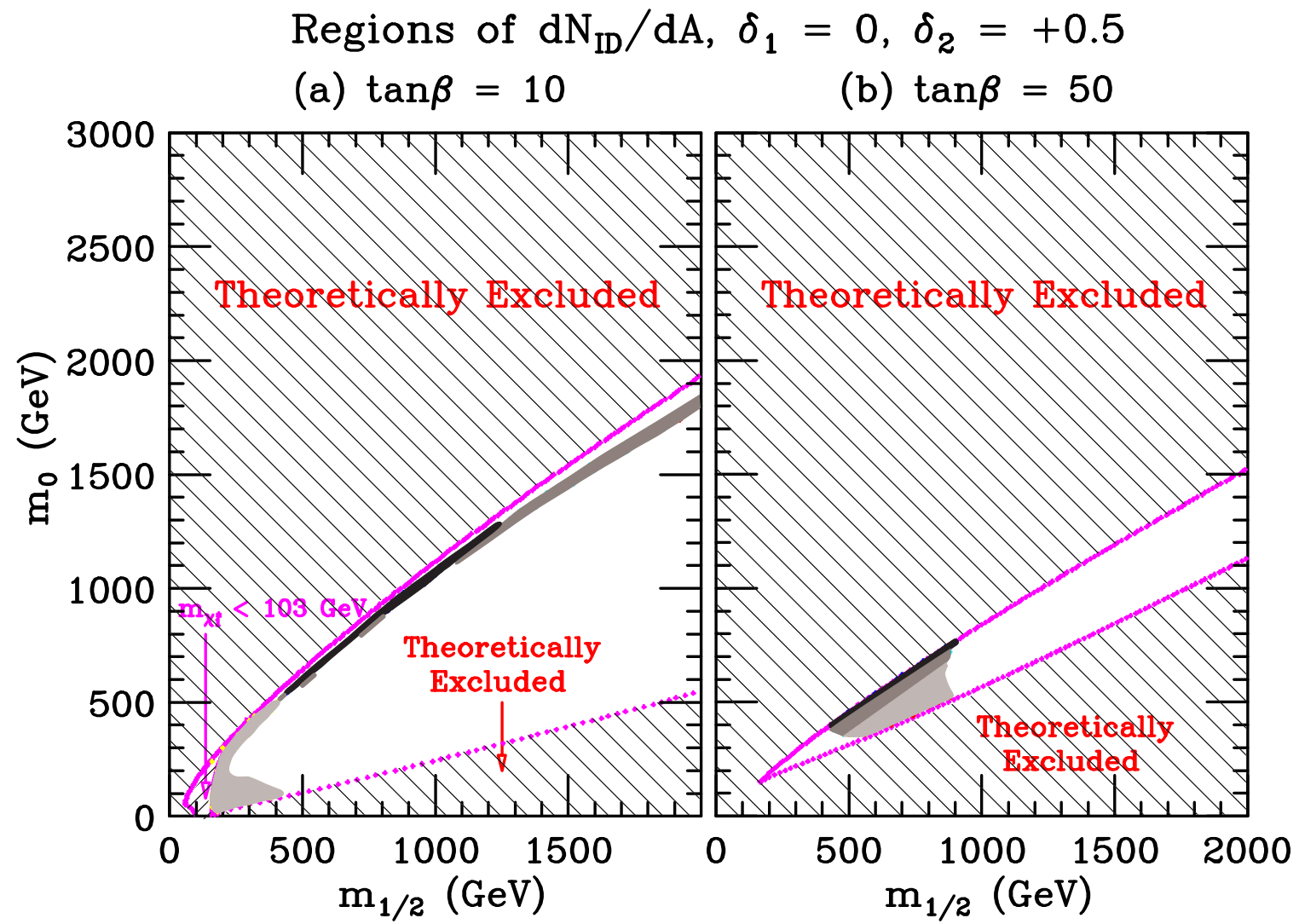

FIG. 17. Regions of indirect detection rate of the neutralino dark matter in the $\left(m_{1 / 2}, m_{0}\right)$ plane of a non-universal SUGRA model with $\mu>0, A_{0}=0$ and non-universal boundary conditions $\delta_{1}=0$ and $\delta_{2}=+0.5$, for (a) $\tan \beta=10$ and (b) $\tan \beta=50$. The region with dark shading has $d N_{\mathrm{ID}} / d A>10$; the region with light shading has $d N_{\mathrm{ID}} / d A<1$; and the region with intermediate shading has $10>d N_{\mathrm{ID}} / d A>1$ (event $/ \mathrm{km}^{2} /$ year). The blank regions do not have a cosmologically interesting relic density $\left(0.05 \leq \Omega_{\chi_{1}^{0}} \leq 0.3\right)$ for the neutralino dark matter. Also shown are the parts of the parameter space (i) excluded by theoretical requirements, or (ii) excluded by the chargino search at LEP 2. 


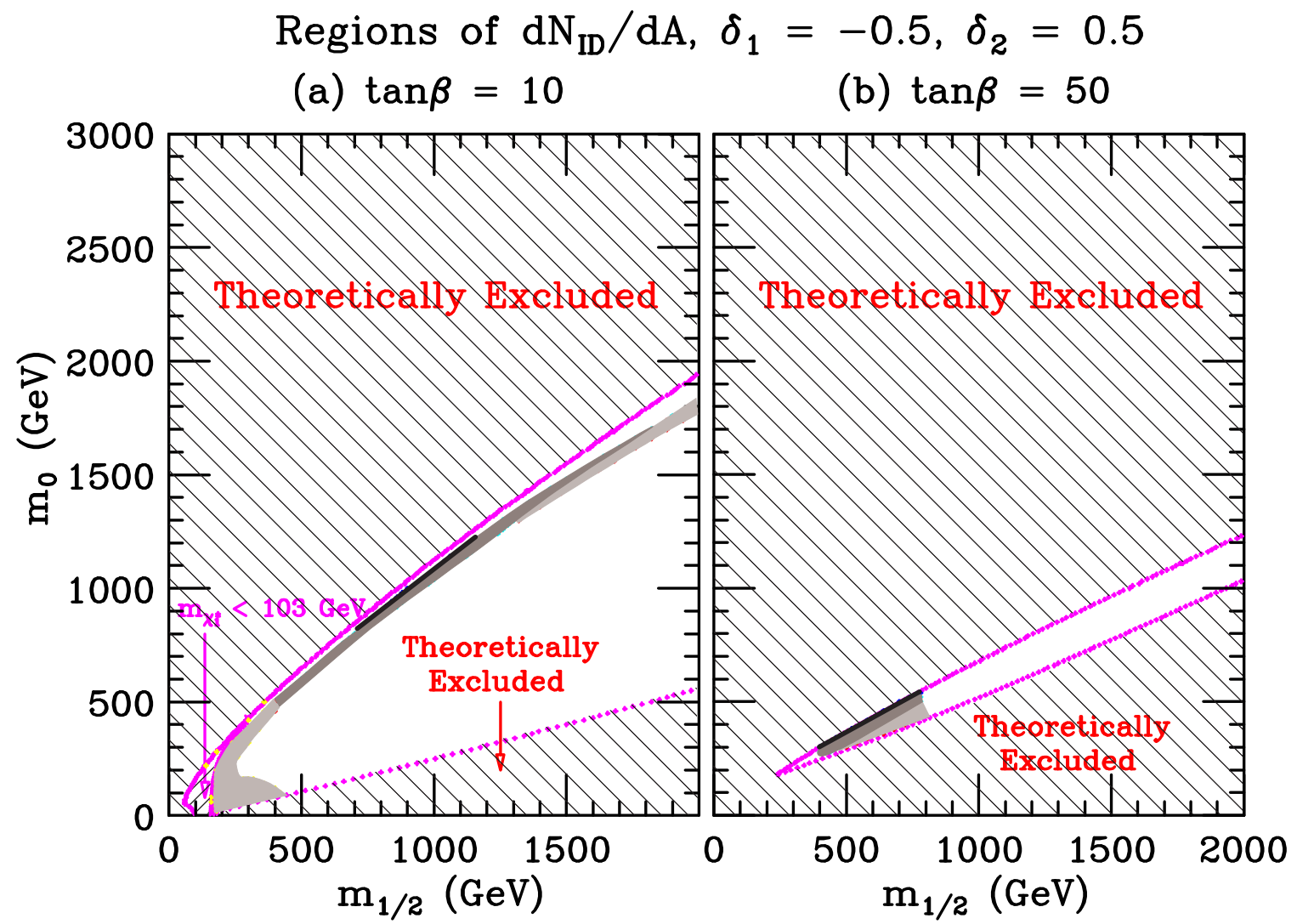

FIG. 18. Regions of indirect detection rate of the neutralino dark matter in the $\left(m_{1 / 2}, m_{0}\right)$ plane of a non-universal SUGRA model with $\mu>0, A_{0}=0$ and non-universal boundary conditions $\delta_{1}=-0.5$ and $\delta_{2}=+0.5$, for (a) $\tan \beta=10$ and (b) $\tan \beta=50$. The region with dark shading has $d N_{\mathrm{ID}} / d A>10$; the region with light shading has $d N_{\mathrm{ID}} / d A<1$; and the region with intermediate shading has $10>d N_{\mathrm{ID}} / d A>1$ (event $/ \mathrm{km}^{2} /$ year). The blank regions do not have a cosmologically interesting relic density $\left(0.05 \leq \Omega_{\chi_{1}^{0}} \leq 0.3\right)$ for the neutralino dark matter. Also shown are the parts of the parameter space (i) excluded by theoretical requirements, or (ii) excluded by the chargino search at LEP 2. 